TRANSACTIONS OF THE

AMERICAN MATHEMATICAL SOCIETY

Volume 362, Number 2, February 2010, Pages 815-846

S 0002-9947(09)04883-1

Article electronically published on September 18, 2009

\title{
REPRESENTATION THEORY OF JORDAN SUPERALGEBRAS I
}

\author{
CONSUELO MARTÍNEZ AND EFIM ZELMANOV
}

\begin{abstract}
We complete the classification of irreducible bimodules over simple finite dimensional Jordan superalgebras.
\end{abstract}

\section{INTRODUCTION}

Throughout this paper all algebras are considered over a ground field $F$ that is algebraically closed of characteristic zero.

A (linear) Jordan algebra is a vector space $J$ with a binary bilinear operation $(x, y) \rightarrow x y$ satisfying the following identities:

$$
\begin{aligned}
x y & =y x, \\
\left(x^{2} y\right) x & =x^{2}(y x) .
\end{aligned}
$$

Let $V$ be an $F$-vector space of countable dimension and let $G=G(V)$ denote the Grassmann (or exterior) algebra over $V$; that is, the quotient of the tensor algebra over the ideal generated by the symmetric tensors. Then $G(V)$ is a $\mathbb{Z} / 2 \mathbb{Z}$-graded algebra, $G(V)=G(V)_{\overline{0}}+G(V)_{\overline{1}}$. Its even part $G(V)_{\overline{0}}$ is the linear span of all tensors of even length, and the odd part $G(V)_{\overline{1}}$ is the linear span of all tensors of odd length.

If $\mathcal{V}$ is a variety of algebras defined by homogeneous identities (see 1, 19]), a superalgebra $A=A_{\overline{0}}+A_{\overline{1}}$ is a $\mathcal{V}$ - superalgebra if its Grassmann enveloping algebra $G(A)=A_{\overline{0}} \otimes G(V)_{\overline{0}}+A_{\overline{1}} \otimes G(V)_{\overline{1}}$ lies in $\mathcal{V}$.

Given an element $a \in A_{\overline{0}} \cup A_{\overline{1}},|a|$ denotes its parity (0 or 1 ).

Thus, a Jordan superalgebra is a $\mathbb{Z} / 2 \mathbb{Z}$-graded algebra $J=J_{\overline{0}}+J_{\overline{1}}$ satisfying the graded identities

$$
x y=(-1)^{|x||y|} y x
$$

(supercommutativity) and

$$
\begin{gathered}
((x y) z) t+(-1)^{|y||z|+|y||t|+|z||t|}((x t) z) y+(-1)^{|x||y|+|x||z|+|x||t|+|z||t|}((y t) z) x \\
=(x y)(z t)+(-1)^{|y||z|}(x z)(y t)+(-1)^{|t|(|y|+|z|)}(x t)(y z) .
\end{gathered}
$$

Received by the editors December 19, 2007.

2000 Mathematics Subject Classification. Primary 17C70; Secondary 17C55, 17B10, 17B60.

Key words and phrases. Jordan superalgebra, Jordan bimodule.

The first author was partially supported by MTM 2007-67884-C04-01 and FICYT IB-05-186.

The second author was partially supported by the NSF grants FRG DMS-0455906 and DMS0500568. 
Examples. I) $A=M_{m+n}(F), A_{\overline{0}}=\left(\begin{array}{cc}\star & 0 \\ 0 & \star\end{array}\right), A_{\overline{1}}=\left(\begin{array}{cc}0 & \star \\ \star & 0\end{array}\right)$ and

II) $A=Q(n)=\left(\begin{array}{ll}a & b \\ b & a\end{array}\right) \mid a, b \in M_{n}(F)$ are associative superalgebras.

C.T.C. Wall [18] proved that every associative simple finite-dimensional superalgebra over the algebraically closed field $F$ is isomorphic to one of them.

III) Let $A$ be an associative (super)algebra. The new operation

$$
a \cdot b=\frac{1}{2}\left(a b+(-1)^{|a||b|} b a\right)
$$

defines a structure of a Jordan (super)algebra on $A$. We will denote this Jordan (super)algebra as $A^{(+)}$. The Lie superalgebra $\left(A,[a, b]=a b-(-1)^{|a||b|} b a\right)$ will be denoted as $A^{(-)}$.

In this way we get the first examples of Jordan simple finite-dimensional superalgebras, applying III) to the associative superalgebras I) and II):

1) $M_{m+n}^{(+)}(F), m \geq 1, n \geq 1$;

2) $Q(n)^{(+)}, n \geq 2$.

If $A$ is an associative superalgebra and $\star: A \rightarrow A$ is a superinvolution, that is, $\left(a^{\star}\right)^{\star}=a,(a b)^{\star}=(-1)^{|a||b|} b^{\star} a^{\star}$, then the set of symmetric elements $H(A, \star)$ is a (Jordan) subsuperalgebra of $A^{(+)}$. Similarly the set of skewsymmetric elements $\operatorname{Skew}(A, \star)$ is a Lie subsuperalgebra of $A^{(-)}$.

The following two subalgebras of $M_{m+n}^{(+)}$are of this type:

3) Let $I_{n}, I_{m}$ be the identity matrices, $t$ the transposition and $U=-U^{t}=$ $-U^{-1}=\left(\begin{array}{cc}0 & -I_{m} \\ I_{m} & 0\end{array}\right)$. Then $\star: M_{n+2 m}(F) \rightarrow M_{n+2 m}(F)$ given by

$$
\left(\begin{array}{ll}
a & b \\
c & d
\end{array}\right)^{\star}=\left(\begin{array}{cc}
I_{n} & 0 \\
0 & U
\end{array}\right)\left(\begin{array}{cc}
a^{t} & -c^{t} \\
b^{t} & d^{t}
\end{array}\right)\left(\begin{array}{cc}
I_{n} & 0 \\
0 & U^{-1}
\end{array}\right)
$$

is a superinvolution.

We will refer to $O S P_{n, 2 m}(F)=\operatorname{Skew}\left(M_{n+2 m}(F), \star\right)$ and $J_{o s p}, 2 m(F)=$ $H\left(M_{n+2 m}(F), \star\right)$ as the Lie and the Jordan orthosymplectic superalgebras respectively.

4) The associative superalgebra $M_{n+n}(F)$ has another superinvolution:

$$
\left(\begin{array}{ll}
a & b \\
c & d
\end{array}\right)^{\sigma}=\left(\begin{array}{cc}
d^{t} & -b^{t} \\
c^{t} & a^{t}
\end{array}\right)
$$

The Lie superalgebra of skewsymmetric elements and the Jordan superalgebra of symmetric elements are denoted by $P_{n}(F)$ and $J P_{n}(F)$ respectively.

5) The 3-dimensional Kaplansky superalgebra, $K_{3}=F e+(F x+F y)$, with the multiplication $e^{2}=e, e x=\frac{1}{2} x, e y=\frac{1}{2} y,[x, y]=e$ is not unital.

6) The 1-parametric family of 4-dimensional superalgebras $D_{t}$ is defined as $D_{t}=$ $\left(F e_{1}+F e_{2}\right)+(F x+F y)$ with the product $e_{i}^{2}=e_{i}, e_{1} e_{2}=0, e_{i} x=\frac{1}{2} x, e_{i} y=$ $\frac{1}{2} y, x y=e_{1}+t e_{2}, t \in F, i=1,2$.

The superalgebra $D_{t}$ is simple if $t \neq 0$. In the case $t=-1$, the superalgebra $D_{-1}$ is isomorphic to $M_{1+1}(F)^{(+)}$. 
7) Let $V=V_{\overline{0}}+V_{\overline{1}}$ be a $\mathbb{Z} / 2 \mathbb{Z}$-graded vector space with a superform $(\mid): V \times V \rightarrow$ $F$ which is symmetric on $V_{\overline{0}}$, skewsymmetric in $V_{\overline{1}}$ and $\left(V_{\overline{0}} \mid V_{\overline{1}}\right)=(0)=\left(V_{\overline{1}} \mid V_{\overline{0}}\right)$.

The superalgebra $J=F 1+V=\left(F 1+V_{\overline{0}}\right)+V_{\overline{1}}$ is Jordan.

8) V. Kac introduced the 10-dimensional superalgebra $K_{10}$ that is related (via the Tits-Kantor-Koecher construction) to the exceptional 40-dimensional Lie superalgebra. It was proved in [14 that this superalgebra is not a homomorphic image of a special Jordan superalgebra.

9) I. Kantor defined a Jordan superalgebra structure in the finite-dimensional Grassmann algebra generated by $e_{1}, \ldots, e_{n}$. These simple Jordan superalgebras have nonsemisimple even parts and are not special, though they are homomorphic images of special algebras $(8])$.

V. Kac 2] (see also I. Kantor [3]) proved that every simple finite-dimensional Jordan superalgebra over $F$ is isomorphic to one of the superalgebras $M_{n+m}(F)^{(+)}$, $Q_{n}(F)^{(+)}, J_{o s p}, 2 m(F), J P_{n}(F)$, a superalgebra of a superform, $K_{3}, D_{t}, K_{10}$, or a Kantor superalgebra.

If $J$ is a Jordan (super)algebra, a Jordan bimodule $V$ over $J$ is a $\mathbb{Z} / 2 \mathbb{Z}$-graded vector space with operations $V \times J \rightarrow V, J \times V \rightarrow V$ such that the split null extension $V+J$ is a Jordan (super)algebra (see [1]). Recall that the split null extension is the direct sum of vector spaces $V+J$ with the operation that extends the multiplication of $J$ and the action of $J$ on $V$, while the product of two arbitrary elements in $V$ is zero.

If $V=V_{\overline{0}}+V_{\overline{1}}$ is a Jordan bimodule over a Jordan superalgebra $J$, then the bimodule $V^{o p}=V_{\overline{1}}^{o p}+V_{\overline{0}}^{o p}$, where the parity of the subspace $V_{\bar{i}}^{o p}$ is different from $\bar{i}$ and the action of $J$ is defined via

$$
a v^{o p}=(-1)^{|a|}(a v)^{o p}, \quad v^{o p} a=(v a)^{o p}
$$

for arbitrary $a \in J, v \in V$ is also a Jordan bimodule, which is denoted as $V^{o p}$ and called the opposite of the bimodule $V$.

Given an arbitrary set $X$, there is a unique free $J$-bimodule $V(X)$ over the set of free generators $X$. If $V^{\prime}$ is a $J$-bimodule, then an arbitrary map $X \rightarrow V^{\prime}$ uniquely extends to a homomorphism of bimodules $V(X) \rightarrow V^{\prime}$.

Let $X$ be a set consisting of one element. For an element $a \in J$ let $R_{V(X)}(a)$ denote the multiplication operator $R_{V(X)}(a): V(X) \rightarrow V(X), v \rightarrow v a$.

The subalgebra $U(J)$ of the algebra of all linear transformations of $V(X)$ generated by the operators $R_{V(X)}(a), a \in J$, is called multiplicative enveloping of $J$.

Every Jordan bimodule over $J$ is a $U(J)$-right module, and conversely.

Let's denote $D(x, y)=R(x) R(y)-(-1)^{|x||y|} R(y) R(x), V(x, y)=D(x, y)+$ $R(x y), U(x, y)=R(x) R(y)+(-1)^{|x||y|} R(y) R(x)-R(x y)$ and $\{x, y, z\}=y U(x, z)=$ $(x y) z+x(y z)-(-1)^{|x||y|} y(x z)$, the Jordan triple product.

We will need the following identities which hold in all Jordan superalgebras:

(1) $D(x, y)$ is a superderivation,

(2) $D(x y, z)=D(x, y z)+(-1)^{|x||y|} D(y, x z)$,

(3) $R(x) U(y, z)+(-1)^{|x|(|y|+|z|)} U(y, z) R(x)=U(x y, z)+(-1)^{|x||y|} U(y, x z)$,

(4) $R(x) R(y) R(z)=\frac{1}{2}\left(-(-1)^{|y||z|} R((x z) y)+R(x y) R(z)\right.$

$$
\begin{gathered}
+(-1)^{|z||y|} R(x z) R(y)+(-1)^{|x|(|y|+|z|)} R(y z) R(x)+R(x) D(y, z) \\
\left.+(-1)^{|z||y|} D(x, z) R(y)+(-1)^{|z|(|x|+y \mid)} R(z) D(x, y)\right),
\end{gathered}
$$

(5) $U\left(x_{1}, x_{2}\right) U\left(y_{1}, y_{2}\right)= \pm V\left(x_{1}, y_{1}\right) V\left(x_{2}, y_{2}\right) \pm V\left(x_{1}, y_{2}\right) V\left(x_{2}, y_{1}\right)$

$$
\pm 2 V\left(x_{1}, x_{2} U\left(y_{1}, y_{2}\right)\right) \text {. }
$$


In [1], N. Jacobson developed the representation theory of semisimple finitedimensional Jordan algebras. He proved that:

i) if $J$ is a finite-dimensional Jordan algebra, then $\operatorname{dim}_{F} U(J)<\infty$,

ii) if $J$ is a finite-dimensional semisimple Jordan algebra, then $U(J)$ is semisimple as well. In particular, all bimodules over $J$ are completely reducible.

Moreover, he determined all irreducible bimodules over simple finite-dimensional Jordan algebras.

Let $e$ be the identity of $J$ and let $V=\{e, V, e\}+\{1-e, V, e\}+\{1-e, V, 1-e\}$ be the Peirce decomposition.

If $V(X)$ is a free Jordan bimodule over $J$, then $V_{1}(X)=\{e, V(X), e\}$ is the free unital bimodule over $J$. An arbitrary map from $X$ to a unital Jordan bimodule $V_{1}$ uniquely extends to a homomorphism of bimodules $V_{1}(X) \rightarrow V_{1}$. We call the associative algebra $U_{1}(J)$ generated by the linear transformations $R_{V_{1}(X)}(a): V_{1}(X) \rightarrow$ $V_{1}(X), v \rightarrow v a$, the unital (multiplicative) enveloping algebra of $J$.

A bimodule $V$ over $J$ is said to be one-sided if $\{J, V, J\}=(0)$. In this case, the mapping $a \rightarrow R_{V}(a) \in \operatorname{End}_{F}(V)$ is a homomorphism of $J$ into $\operatorname{End}_{F}(V)^{(+)}$.

The subbimodule $W(X)=\{e, V(X), 1-e\}+\{1-e, V(X), 1-e\}$ is the universal one-sided bimodule over $J$. The associative subalgebra $S(X)$ of $\operatorname{End}_{F}(W(X))$ generated by $R_{W(X)}(J)$ is the universal (associative) enveloping algebra of $J$ (see [1]).

As in [1, Th. 15, p. 103], $U(J) \simeq U_{1}(J) \oplus S(J)$.

If $J=J^{\prime} \oplus J^{\prime \prime}$, then $U_{1}(J) \simeq U_{1}\left(J^{\prime}\right) \oplus U_{1}\left(J^{\prime \prime}\right) \oplus\left(U_{1}\left(J^{\prime}\right) \otimes U_{1}\left(J^{\prime \prime}\right)\right)$ (see [1]).

In [1] we classified one-sided finite-dimensional bimodules over simple finitedimensional Jordan superalgebras.

The purpose of this paper is the classification of irreducible unital finite-dimensional bimodules over all simple finite-dimensional Jordan superalgebras. Recall that the irreducible bimodules over the exceptional 10-dimensional Kac superalgebra $K_{10}$ were classified by A. S. Shtern (see [16]). Irreducible and even indecomposible bimodules over $D_{t}$ were classified in [12 (see also [17). Classification of irreducible finite-dimensional bimodules over $Q(n)^{(+)}$and over $J P_{n}(F)$ is done in 9. In the forthcoming paper [13] we will treat the case of indecomposible modules.

\section{Contents}

1. Tits-Kantor-Koecher construction.

2. Root graded modules.

3. Irreducible bimodules over $J P(n), n \geq 2$.

4. Irreducible bimodules over $M_{m+n}(F)^{(+)}, m \geq n, m+n \geq 3$.

5. Unital irreducible bimodules over $M_{1+1}(F)^{(+)}$.

6. Irreducible bimodules over $\operatorname{Josp}(m, 2 r)$.

7. Irreducible bimodules over Jordan superalgebras of superforms.

8. Jordan superalgebras of rank $\geq 3$.

In Chapters 1 and 2 we relate irreducible representations of Jordan superalgebras to root graded representations of the corresponding Tits-Kantor-Koecher Lie superalgebras.

In Chapters 3-7 some irreducible representations for each of the types of Jordan superalgebras are constructed. The subsequent analysis of highest weights of the corresponding root graded modules shows that these lists are complete. 
In Chapter 8 we prove that for a simple finite-dimensional unital Jordan superalgebra $J$, which contains 3 pairwise orthogonal idempotents in its even part, the universal multiplicative enveloping algebra $U(J)$ is finite-dimensional and semisimple. Thus all finitely generated $J$-bimodules are finite-dimensional and completely reducible.

\section{Tits-Kantor-Koecher COnstruction}

Definition $1.1([6])$. A Jordan (super)pair $P=\left(P^{-}, P^{+}\right)$is a pair of vector (super)spaces with a pair of trilinear operations

$$
\{,,\}: P^{-} \times P^{+} \times P^{-} \rightarrow P^{-}, \quad\{,,\}: P^{+} \times P^{-} \times P^{+} \rightarrow P^{+}
$$

that satisfies the following identities:

(P.1) $\left\{x^{\sigma}, y^{-\sigma},\left\{x^{\sigma}, z^{-\sigma}, x^{\sigma}\right\}\right\}=\left\{x^{\sigma},\left\{y^{-\sigma}, x^{\sigma}, z^{-\sigma}\right\}, x^{\sigma}\right\}$,

(P.2) $\left\{\left\{x^{\sigma}, y^{-\sigma}, x^{\sigma}\right\}, y^{-\sigma}, u^{\sigma}\right\}=\left\{x^{\sigma},\left\{y^{-\sigma}, x^{\sigma}, y^{-\sigma}\right\}, u^{\sigma}\right\}$,

(P.3) $\left\{\left\{x^{\sigma}, y^{-\sigma}, x^{\sigma}\right\}, z^{-\sigma},\left\{x^{\sigma}, y^{-\sigma}, x^{\sigma}\right\}\right\}=\left\{x^{\sigma},\left\{y^{-\sigma},\left\{x^{\sigma}, z^{-\sigma}, x^{\sigma}\right\}, y^{-\sigma}\right\}, x^{\sigma}\right\}$,

for every $x^{\sigma}, u^{\sigma} \in P^{\sigma}, y^{-\sigma}, z^{-\sigma} \in P^{-\sigma}, \sigma= \pm$ (see []. $)$.

Let $L=L_{-1}+L_{0}+L_{1}$ be a $\mathbb{Z}$-graded Lie (super)-algebra. Then $\left(L_{-1}, L_{1}\right)$ is a Jordan (super)pair with respect to the trilinear operations $\left\{x^{\sigma}, y^{-\sigma}, z^{\sigma}\right\}=$ $\left[\left[x^{\sigma}, y^{-\sigma}\right], z^{\sigma}\right] ; x^{\sigma}, z^{\sigma} \in L_{\sigma 1}, y^{-\sigma} \in L_{-\sigma 1}, \sigma= \pm$.

Theorem 1.2. For an arbitrary Jordan (super) pair $P=\left(P^{-}, P^{+}\right)$, there exists a unique $Z$-graded Lie (super) algebra $K=K_{-1}+K_{0}+K_{1}$ such that $\left(K_{-1}, K_{1}\right) \simeq P$, $K_{0}=\left[K_{-1}, K_{1}\right]$, and for every 3-graded Lie (super)algebra $L=L_{-1}+L_{0}+L_{1}$, an arbitrary homomorphism of the Jordan pairs $P \rightarrow\left(L_{-1}, L_{1}\right)$ uniquely extends to a homomorphism of Lie (super)algebras $K \rightarrow L$.

The uniqueness is obvious. Let us prove the existence. Choose bases $\left\{e_{i}^{+}\right\}_{i}$ of $P^{+}$and $\left\{e_{j}^{-}\right\}_{j}$ of $P^{-}$, and consider the multiplication table

$$
\begin{aligned}
& \left\{e_{i}^{+}, e_{j}^{-}, e_{k}^{+}\right\}=\sum_{t} \gamma_{i j k}^{t} e_{t}^{+}, \quad \gamma_{i j k}^{t} \in F, \\
& \left\{e_{j}^{-}, e_{i}^{+}, e_{q}^{-}\right\}=\sum_{s} \xi_{j i q}^{s} e_{s}^{-}, \quad \xi_{j i q}^{s} \in F .
\end{aligned}
$$

Define a Lie (super)algebra $K=K(P)$ by generators $\left\{x_{i}^{+}, x_{j}^{-}\right\}$and relations

$$
\begin{gathered}
{\left[\left[x_{i}^{+}, x_{j}^{-}\right], x_{k}^{+}\right]=\sum_{t} \gamma_{i j k}^{t} x_{t}^{+},} \\
{\left[\left[x_{j}^{-}, x_{i}^{+}\right], x_{q}^{-}\right]=\sum_{s} \xi_{j i q}^{s} x_{s}^{-},} \\
{\left[x_{i}^{+}, x_{k}^{+}\right]=\left[x_{j}^{-}, x_{q}^{-}\right]=0 .}
\end{gathered}
$$

The resulting algebra $K$ is $\mathbb{Z}$-graded (let $\operatorname{deg} x_{i}^{+}=1, \operatorname{deg} x_{j}^{-}=-1$ ). Moreover, $K$ is spanned by $x_{i}^{+}, x_{j}^{-}$and $\left[x_{i}^{+}, x_{j}^{-}\right]$, which implies that $K_{i}=(0)$ for $|i| \geq 2$.

It is easy to see that $K$ has the required universal property.

We will refer to $K=K(P)$ as the TKK-construction of the pair $P$.

If $J$ is a Jordan superalgebra, then $\left(J^{-}, J^{+}\right)$is a Jordan superpair. The Lie superalgebra $K=K\left(J^{-}, J^{+}\right)$is called the TKK-construction of $J$. 


\section{Root GRADED MODULES}

Let $J=J_{\overline{0}}+J_{\overline{1}}$ be a simple finite-dimensional Jordan superalgebra. Let's consider $L=K(J)$ as its TKK-construction.

If $V$ is a Jordan bimodule over $J$, then the null extension $V+J$ is a Jordan superalgebra, so we can consider its TKK Lie superalgebra $K(V+J)=$ $\left(V^{-}+J^{-}\right)+\left[V^{-}+J^{-}, V^{+}+J^{+}\right]+\left(V^{+}+J^{+}\right)$.

Denote $K(V)=V^{-}+\left[V^{-}, J^{+}\right]+\left[J^{-}, V^{+}\right]+V^{+} \leq K(V+J)$. Then $K(V)$ is a Lie module over the subalgebra $J^{-}+\left[J^{-}, J^{+}\right]+J^{+}$which is isomorphic to $K(J)$.

Let $W$ be the maximal $K(J)$-submodule, which is contained in $K(V)_{0}=\left[V^{-}, J^{+}\right]$ $+\left[J^{-}, V^{+}\right]$. Let $\bar{K}(V)=K(V) / W$.

Lemma 2.1. Let $J$ be a unital Jordan (super)algebra and let $V_{1}, V_{2}$ be two unital Jordan J-bimodules. The following assertions are equivalent:

(1) $V_{1} \simeq V_{2}$,

(2) $K\left(V_{1}\right) \simeq K\left(V_{2}\right)$,

(3) $\bar{K}\left(V_{1}\right) \simeq \bar{K}\left(V_{2}\right)$.

Proof. Clearly $(1) \Rightarrow(2) \Rightarrow(3)$. Let us show that $(3) \Rightarrow(1)$.

Let $\varphi: \bar{K}\left(V_{1}\right) \rightarrow \bar{K}\left(V_{2}\right)$ be a $K(J)$-module isomorphism. Let $e$ be the identity element of $J$. Then $s l_{2}(F) \simeq F e^{-}+F\left[e^{-}, e^{+}\right]+F e^{+} \leq K(J)$, and $V_{i}^{+}$is the eigenspace of $\bar{K}\left(V_{i}\right)$ with respect to ad $\left(\left[e^{-}, e^{+}\right]\right)$, which corresponds to the eigenvalue -2 . This implies that $\varphi\left(V_{1}^{+}\right)=V_{2}^{+}$. Both spaces $V_{1}^{+}, V_{2}^{+}$are identified with $V_{1}, V_{2}$ respectively. Let us show that $\varphi_{\mid V_{1}^{+}}$is a bimodule isomorphism.

Choose $a \in J, v_{1} \in V_{1}$. Let $\varphi\left(v_{1}^{+}\right)=v_{2}^{+} \in V_{2}^{+}$. The element $\left[\left[a^{+}, e^{-}\right], v_{1}^{+}\right]$ is mapped by $\varphi$ to $\left[\left[a^{+}, e^{-}\right], \varphi\left(v_{1}^{+}\right)\right]$, which is identified with $a v_{2}$. The lemma is proved.

Lemma 2.2. For a unital Jordan bimodule $V$ over a unital Jordan (super)algebra $J$, the following assertions are equivalent:

(1) $V$ is an irreducible $J$-bimodule,

(2) $\bar{K}(V)$ is an irreducible $K(J)$-module

Proof. (1) $\Rightarrow(2)$ Let $W$ be a $K(J)$-subbimodule of $\bar{K}(V)$. Choose $w \in W, w=$ $w_{-2}+w_{0}+w_{2},\left[\left[e^{-}, e^{+}\right], w_{i}\right]=i w_{i}$. Using a Vandermonde type argument we get $w_{i} \in W, i=-2,0,2$. Hence $W=\left(W \cap \bar{K}(V)_{-2}\right)+\left(W \cap \bar{K}(V)_{0}\right)+\left(W \cap \bar{K}(V)_{2}\right)$.

Suppose that $W \cap \bar{K}(V)_{-2} \neq(0)$. The subspace $\bar{K}(V)_{-2}$ is identified with $V$. It is easy to see that the intersection of $V$ with $W$ is a subbimodule of $V$. Hence $\bar{K}(V)_{-2} \subseteq W$.

Since $\bar{K}(V)_{2}=\left[e^{+},\left[e^{+}, \bar{K}(V)_{-2}\right]\right]$ it follows that $\bar{K}(V)_{2} \subseteq W$ as well, and therefore $\bar{K}(V)=W$.

If $W \subseteq \bar{K}(V)_{0}$ then $W=(0)$ since $\bar{K}(V)$ does not contain nonzero submodules in $\bar{K}(V)_{0}$.

$(2) \Rightarrow(1)$ Now suppose that the $K(J)$-module $\bar{K}(V)$ is irreducible. If $V^{\prime}$ is a proper subbimodule of $V$, then $V^{\prime-}+\left[V^{\prime-}, J^{+}\right]+\left[J^{-}, V^{\prime+}\right]+V^{\prime+}$ is a proper $K(J)$-subbimodule of $\bar{K}(V)$. The lemma is proved.

Lemma 2.3. Let $J$ be a simple finite-dimensional Jordan superalgebra, $L=K(J)$.

(1) Suppose that both $L_{\overline{0}}$ and $J_{\overline{0}}$ are semisimple and $J_{\overline{0}}$ has the same number of simple summands as $L_{\overline{0}}$. Then $L_{\overline{0}}=K\left(J_{\overline{0}}\right)$. 
(2) Suppose now that $L_{\overline{0}} /$ Center is semisimple and that $J_{\overline{0}}$ is semisimple and has the same number of simple summands as $L_{\overline{0}} /$ Center. Then $\left[L_{\overline{0}}, L_{\overline{0}}\right]=$ $\left[K\left(J_{\overline{0}}\right), K\left(J_{\overline{0}}\right)\right]$.

Proof. (1) We have $L_{\overline{0}}=J_{\overline{0}}^{-}+\left[J_{\overline{0}}^{-}, J_{\overline{0}}^{+}\right]+\left[J_{\overline{1}}^{-}, J_{\overline{1}}^{+}\right]+J_{\overline{0}}^{+}$. This implies that $J_{\overline{0}}^{-}+$ $\left[J_{\overline{0}}^{-}, J_{\overline{0}}^{+}\right]+J_{\overline{0}}^{+}=K\left(J_{\overline{0}}\right)$ is an ideal of $L_{\overline{0}}$. Let $J_{\overline{0}}$ be a direct sum of s simple summands, $J_{\overline{0}}=J_{\overline{0}}^{(1)} \oplus \cdots \oplus J_{\overline{0}}^{(s)}$. Then $K\left(J_{\overline{0}}\right)=K\left(J_{\overline{0}}^{(1)}\right) \oplus \cdots \oplus K\left(J_{\overline{0}}^{(s)}\right)$. Since $L_{\overline{0}}$ is semisimple and $L_{\overline{0}}$ and $K\left(J_{\overline{0}}\right)$ have the same number of simple summands, it follows that $K\left(J_{\overline{0}}\right)=L_{\overline{0}}$.

(2) Arguing as above, we get

$$
K\left(J_{\overline{0}}\right)+\operatorname{Center}\left(L_{\overline{0}}\right) / \operatorname{Center}\left(L_{\overline{0}}\right)=L_{\overline{0}} / \operatorname{Center}\left(L_{\overline{0}}\right),
$$

so $K\left(J_{\overline{0}}\right)+\operatorname{Center}\left(L_{\overline{0}}\right)=L_{\overline{0}}$. This implies the result and proves the lemma.

Remark 2.4. The superalgebras $\operatorname{Josp}(n, 2 m)$ and $J P(n), n \geq 3$ satisfy condition (1). The superalgebras $M_{n+m}(F)^{(+)}, Q_{n}(F)^{(+)}$and $J P(2)$ satisfy condition (2).

Now we will define root graded modules.

Let $J$ be a simple finite-dimensional Jordan superalgebra of one of the types $J o s p(n, 2 m), J P(n), n \geq 2, M_{n+m}(F)^{(+)}, Q(n)^{(+)}, L=K(J)$ its universal TKKconstruction and $\mathcal{H}$ a Cartan subalgebra of $\left[L_{\overline{0}}, L_{\overline{0}}\right]$. As we have just seen, $\left[L_{\overline{0}}, L_{\overline{0}}\right]=$ $\left[K\left(J_{\overline{0}}\right), K\left(J_{\overline{0}}\right)\right]$.

Let $\left\{V_{i}\right\}$ be the family of all finite-dimensional irreducible unital bimodules over $J_{\overline{0}}$. Then $\left\{K\left(V_{i}\right)\right\}$ are modules over $K\left(J_{\overline{0}}\right)$. Let $\Delta(J)$ be the set of all nonzero weights of all modules $K\left(V_{i}\right)$ with respect to $\mathcal{H}$.

Definition 2.5. A module $W$ over $L$ is said to be root graded if

(1) the action of $\mathcal{H}$ on $W$ is diagonalizable,

(2) $W_{\alpha} \neq(0), \alpha \neq 0$, implies $\alpha \in \Delta(J)$,

(3) $W$ is generated (as a module) by $\sum_{\alpha \neq 0} W_{\alpha}$.

Lemma 2.6. Let $V$ be a unital bimodule over a simple finite-dimensional unital Jordan superalgebra $J$ of one of the types $J P(n), Q(n)^{(+)}, M_{m+n}(F)^{(+)}$, or $J \operatorname{Josp}(n, 2 m)$, and let $L=K(J)$. Then the $L$-module $K(V)$ is root-graded

Proof. Let's view $V$ as a bimodule over the semisimple finite-dimensional Jordan algebra $J_{\overline{0}}, V=\sum_{i} V_{i}$, where each $V_{i}$ is an irreducible $J_{\overline{0}}$-bimodule. From the description of unital $J_{\overline{0}}$-bimodules, it follows that the action of $\mathcal{H}, \mathcal{H} \subset K\left(J_{\overline{0}}\right)$, on $K(V)$ is diagonalizable.

Let $0 \neq \alpha \in \mathcal{H}^{\star},\left[V^{-}, J_{\overline{1}}^{+}\right] \neq(0)$, and $v \in\left[V^{-}, J_{\overline{1}}^{+}\right]_{\alpha}$. Then there exists $h \in \mathcal{H}$ such that $[v, h]=\alpha(h) v=\lambda v$, with $\lambda \neq 0$. Hence $v \in\left[\left[V^{-}, J_{\overline{1}}^{+}\right], \mathcal{H}\right] \subseteq$ $\left[\left[V^{-}, J_{\overline{1}}^{+}\right],\left[J_{\overline{0}}^{-}, J_{\overline{0}}^{+}\right]\right] \subseteq\left[V^{-}, J_{\overline{0}}^{+}\right]+\left[V^{+}, J_{\overline{0}}^{-}\right]$.

Similarly, every root space $\left[V^{+}, J_{\overline{1}}^{-}\right]_{\alpha}, 0 \neq \alpha \in \mathcal{H}^{\star}$, lies in $\left[V^{-}, J_{\overline{0}}^{+}\right]+\left[V^{+}, J_{\overline{0}}^{-}\right]$.

Hence $K(V)=K(V)_{0}+V^{-}+V^{+}+\left[V^{-}, J_{\overline{0}}^{+}\right]+\left[V^{+}, J_{\overline{0}}^{-}\right]=K(V)_{0}+\sum_{i} V_{i}^{-}+$ $\sum_{i} V_{i}^{+}+\sum_{i}\left[V_{i}^{-}, J_{\overline{0}}^{+}\right]+\sum_{i}\left[V_{i}^{+}, J_{\overline{0}}^{-}\right]$, where $K(V)_{0}$ is the centralizer of $\mathcal{H}$ in $K(V)$. Hence $K(V)_{\alpha} \neq(0), 0 \neq \alpha \in \mathcal{H}^{*}$, implies $\alpha \in \Delta(J)$.

Since $K(V)$ is generated by $V^{-}, V^{+}$, we conclude that $K(V)$ is generated by $\sum_{\alpha \in \Delta(J)} K(V)_{\alpha}$. The lemma is proved.

Now we will determine $\Delta(J)$ for various root systems. 
I) Case $J P(n)$.

$$
\begin{gathered}
J=J P(n)=\left\{\left(\begin{array}{cc}
a & b \\
c & a^{t}
\end{array}\right) \mid a \in M_{n}(F), b^{t}=-b, c^{t}=c\right\}, \\
K(J)=P(2 n-1)=\left\{\left(\begin{array}{cc}
a & b \\
c & -a^{t}
\end{array}\right) \mid \operatorname{tr}(a)=0, b^{t}=-b, c^{t}=c ; a, b, c \in M_{2 n}(F)\right\}
\end{gathered}
$$

if $n>2$.

The Lie superalgebra $K(J P(2))$ is the universal central cover of the Lie superalgebra $P(3)([10])$.

In this case, $J_{\overline{0}}=M_{n}(F)^{(+)}, K\left(J_{\overline{0}}\right)=s l(2 n)$.

The set of irreducible modules over $J_{\overline{0}}$ consists of the regular bimodule and the four subspaces $\left(\begin{array}{cc}0 & H(n) \\ 0 & 0\end{array}\right),\left(\begin{array}{cc}0 & K(n) \\ 0 & 0\end{array}\right),\left(\begin{array}{cc}0 & 0 \\ H(n) & 0\end{array}\right),\left(\begin{array}{cc}0 & 0 \\ K(n) & 0\end{array}\right)$ of $M_{2 n}(F)$, where $H(n)=\left\{a \in M_{n}(F) \mid a^{t}=a\right\}, K(n)=\left\{a \in M_{2 n}(F) \mid a^{t}=-a\right\}$. The action of $J_{\overline{0}}$ on the last four subspaces is defined as follows. We can identify $J_{\overline{0}}=M_{n}(F)^{+}$with $\left\{\left(\begin{array}{cc}a & 0 \\ 0 & a^{t}\end{array}\right), a \in M_{n}(F)\right\} \subseteq M_{2 n}(F)$. Then $J_{\overline{0}}$ acts via the Jordan multiplication in $M_{2 n}(F)^{(+)}$.

The TKK Lie module of the regular bimodule over $J_{\overline{0}}$ is the regular Lie module over $K\left(J_{\overline{0}}\right)=s l(2 n)$. The algebra $s l(2 n)$ is embeddable into $M_{4 n}(F)$ via $a \rightarrow$ $\left(\begin{array}{cc}a & 0 \\ 0 & -a^{t}\end{array}\right)$. It acts on the subspaces $\left(\begin{array}{cc}0 & H(2 n) \\ 0 & 0\end{array}\right),\left(\begin{array}{cc}0 & K(2 n) \\ 0 & 0\end{array}\right),\left(\begin{array}{cc}0 & 0 \\ H(2 n) & 0\end{array}\right)$, $\left(\begin{array}{cc}0 & 0 \\ K(2 n) & 0\end{array}\right)$ via the commutation in $M_{4 n}(F)$.

These are the TKK Lie modules of the corresponding irreducible $J_{\overline{0}}$-bimodules.

It is easy to see that $\Delta(J)=\left\{0 \neq \pm v_{i} \pm v_{j}, 1 \leq i, j \leq 2 n\right\}$.

II) Case $Q(n)^{(+)}$.

$$
\begin{gathered}
J=Q(n)^{(+)}=\left\{\left(\begin{array}{ll}
a & b \\
b & a
\end{array}\right) \mid a, b \in M_{n}(F)\right\}, n \geq 2, \\
K(J)=Q(2 n-1)^{(-)}=\left\{\left(\begin{array}{ll}
a & b \\
b & a
\end{array}\right) \mid a, b \in M_{2 n}(F)\right\}, \\
J_{0}=M_{n}(F)^{+}, K\left(J_{0}\right)=\operatorname{sl}(2 n) .
\end{gathered}
$$

We get the same $\Delta(J)$ as in the case $P$.

III) Case $J=M_{n+m}(F)^{(+)}, n \geq m, m+n \geq 3$.

$$
K(J)=\left\{\left(\begin{array}{ll}
a & b \\
c & d
\end{array}\right) \mid a \in M_{2 n}(F), d \in M_{2 m}(F), \operatorname{tr}(a)=\operatorname{tr}(d)\right\} .
$$

If $n=m$, then $K(J)$ has a nonzero center.

$$
\begin{gathered}
J_{\overline{0}}=\left\{\left(\begin{array}{ll}
a & 0 \\
0 & b
\end{array}\right)\right\} \simeq M_{m}(F)^{+} \oplus M_{n}(F)^{+}, \\
K\left(J_{\overline{0}}\right)=\operatorname{sl}(2 m) \oplus \operatorname{sl}(2 n) .
\end{gathered}
$$

Unital bimodules over $M_{n}(F)^{(+)}$and $M_{m}(F)^{(+)}$yield the sets of weights $\{0 \neq$ $\left.\pm v_{i} \pm v_{j}, 1 \leq i, j \leq 2 m\right\}$ and $\left\{0 \neq \pm w_{p} \pm w_{q}, 1 \leq p, q \leq 2 n\right\}$.

Let $e_{1}=\left(\begin{array}{cc}I_{m} & 0 \\ 0 & 0\end{array}\right), e_{2}=\left(\begin{array}{cc}0 & 0 \\ 0 & I_{n}\end{array}\right), e_{1}+e_{2}=1$. 
If $V$ is a unital irreducible bimodule over $J_{0}$, then $V=\left\{e_{1}, V, e_{1}\right\}$ or $V=$ $\left\{e_{2}, V, e_{2}\right\}$ or $V=\left\{e_{1}, V, e_{2}\right\}$. In the first case (similarly in the second) $V$ is a bimodule over $M_{m}(F)^{(+)}$(resp. $\left.M_{n}(F)^{(+)}\right)$, so it is one of those mentioned above.

Let $V=\left\{e_{1}, V, e_{2}\right\}$. There are $\leq 4$ irreducible bimodules of this type. Indeed, such a bimodule is a one-sided bimodule over $M_{n}(F)^{(+)}$, a one-sided bimodule over $M_{m}(F)^{(+)}$, and the actions of $M_{n}(F)^{(+)}$and $M_{m}(F)^{(+)}$commute. So, $V$ is a module over the algebra

$$
U\left(M_{m}(F)^{(+)}\right) \otimes U\left(M_{n}(F)^{(+)}\right)=\left(M_{m}(F) \oplus M_{m}(F)^{t}\right) \otimes\left(M_{n}(F) \oplus M_{n}(F)^{t}\right),
$$

which is a sum of 4 isomorphic copies of $M_{m \times n}(F)$.

There are exactly four bimodules that will be listed below:

1) $V_{1}=\left(\begin{array}{cc}0_{m} & M_{m \times n}(F) \\ 0_{n, m} & 0_{n}\end{array}\right)$.

The action is the Jordan multiplication by $\left(\begin{array}{ll}a & 0 \\ 0 & b\end{array}\right)$ :

$$
\left(\begin{array}{ll}
a & 0 \\
0 & b
\end{array}\right) \circ\left(\begin{array}{ll}
0 & x \\
0 & 0
\end{array}\right)=\frac{1}{2}\left(\begin{array}{cc}
0 & a x+x b \\
0 & 0
\end{array}\right) \text {. }
$$

2) $V_{2}=\left(\begin{array}{cc}0 & M_{m \times n}(F) \\ 0 & 0\end{array}\right)$, but now the product is given by

$$
\left(\begin{array}{ll}
a & 0 \\
0 & b
\end{array}\right) \star\left(\begin{array}{ll}
0 & x \\
0 & 0
\end{array}\right)=\frac{1}{2}\left(\begin{array}{cc}
0 & a^{t} x+x b \\
0 & 0
\end{array}\right) .
$$

3) $V_{3}=\left(\begin{array}{cc}0 & M_{m \times n}(F) \\ 0 & 0\end{array}\right)$, and the product is given by

$$
\left(\begin{array}{ll}
a & 0 \\
0 & b
\end{array}\right) \diamond\left(\begin{array}{ll}
0 & x \\
0 & 0
\end{array}\right)=\frac{1}{2}\left(\begin{array}{cc}
0 & a x+x b^{t} \\
0 & 0
\end{array}\right) .
$$

4) $V_{4}=\left(\begin{array}{cc}0 & M_{m \times n}(F) \\ 0 & 0\end{array}\right)$ with the product given by

$$
\left(\begin{array}{ll}
a & 0 \\
0 & b
\end{array}\right) \bullet\left(\begin{array}{ll}
0 & x \\
0 & 0
\end{array}\right)=\frac{1}{2}\left(\begin{array}{cc}
0 & a^{t} x+x b^{t} \\
0 & 0
\end{array}\right) .
$$

Now we will indicate the corresponding Lie modules.

1) $K\left(V_{1}\right)=\left(\begin{array}{cc}0 & M_{2 m \times 2 n}(F) \\ 0 & 0\end{array}\right)$ with the action

$$
\left[\left(\begin{array}{ll}
a & 0 \\
0 & b
\end{array}\right),\left(\begin{array}{ll}
0 & x \\
0 & 0
\end{array}\right)\right]=\left(\begin{array}{cc}
0 & a x-x b \\
0 & 0
\end{array}\right),
$$

$a \in \operatorname{sl}(2 m), b \in \operatorname{sl}(2 n)$, and the set of roots of this module is $\left\{v_{i}-w_{j} \mid 1 \leq i \leq 2 m\right\}$,

2) $K\left(V_{2}\right)=\left(\begin{array}{cc}0 & M_{2 m \times 2 n}(F) \\ 0 & 0\end{array}\right)$ with the action

$$
\left[\left(\begin{array}{cc}
-a^{t} & 0 \\
0 & b
\end{array}\right),\left(\begin{array}{ll}
0 & x \\
0 & 0
\end{array}\right)\right]=\left(\begin{array}{cc}
0 & -a^{t} x-x b \\
0 & 0
\end{array}\right),
$$

and the set of roots of this module is $\left\{-v_{i}-w_{j} \mid 1 \leq i \leq 2 m\right\}$, 
3) $K\left(V_{3}\right)=\left(\begin{array}{cc}0 & M_{2 m \times 2 n}(F) \\ 0 & 0\end{array}\right)$ with the action

$$
\left[\left(\begin{array}{cc}
a & 0 \\
0 & -b^{t}
\end{array}\right),\left(\begin{array}{cc}
0 & x \\
0 & 0
\end{array}\right)\right]=\left(\begin{array}{cc}
0 & a x+x b^{t} \\
0 & 0
\end{array}\right),
$$

with the set of roots $\left\{v_{i}+w_{j} \mid 1 \leq i \leq 2 m\right\}$,

4) $K\left(V_{4}\right)=\left(\begin{array}{cc}0 & M_{2 m \times 2 n}(F) \\ 0 & 0\end{array}\right)$ with the action

$$
\left[\left(\begin{array}{cc}
-a^{t} & 0 \\
0 & -b^{t}
\end{array}\right),\left(\begin{array}{cc}
0 & x \\
0 & 0
\end{array}\right)\right]=\left(\begin{array}{cc}
0 & -a^{t} x+x b^{t} \\
0 & 0
\end{array}\right)
$$

that has the set of roots $\left\{0 \neq-v_{i}+w_{j} \mid 1 \leq i \leq 2 m\right\}$.

Hence $\Delta(J)=\left\{0 \neq \pm v_{i} \pm v_{j}, 0 \neq \pm w_{k} \pm w_{l}, \pm v_{i} \pm w_{k}\right\}$; that is, the set of all integral vectors of length 2 .

IV) Case Josp $(m, n), n$ even. $J=J o s p(m, 2 r)=H(A, *)$, where $A=M_{m+n}(F)$, $n=2 r$ and $*$ is the superinvolution

$$
\left(\begin{array}{ll}
a & b \\
c & d
\end{array}\right)^{*}=Q^{-1}\left(\begin{array}{cc}
a^{t} & -c^{t} \\
b^{t} & d
\end{array}\right) Q
$$

where $Q=\left(\begin{array}{cc}I_{m} & 0 \\ 0 & U_{2 r}\end{array}\right)$ with $U_{2 r}=\left(\begin{array}{cc}0 & -I_{r} \\ I_{r} & 0\end{array}\right)$.

Hence, $J=J o s p(m, 2 r)=\left\{\left(\begin{array}{ll}a & b \\ c & d\end{array}\right) \mid a=a^{t}, d=-U_{2 r} d^{t} U_{2 r}, c=-U_{2 r} b^{t}\right\}$.

$$
J_{\overline{0}}=\left(\begin{array}{cc}
H(m) & 0 \\
0 & H\left(M_{n}(F), \text { Symp }\right)
\end{array}\right)
$$

and $K(J)=O S P(2 m, 4 r), K\left(J_{\overline{0}}\right)=\left(\begin{array}{cc}D_{2 m} & 0 \\ 0 & \text { Symp }_{2 n}\end{array}\right)$.

The bimodules over $J_{\overline{0}}$ are:

i) bimodules over $H(m)$ : the regular bimodule $H(m)$ and the bimodule $K(m)$ with the action $k \cdot h=\frac{1}{2}(k h+h k)$,

ii) bimodules over $H\left(M_{n}(F), S y m p\right)$ : the regular bimodule and the bimodule $\operatorname{Skew}\left(M_{n}(F)\right.$, Symp $)$,

iii) modules over $U\left(H_{m}\right) \otimes U\left(H\left(M_{n}(F)\right.\right.$, Symp $)=M_{m}(F) \otimes M_{n}(F)=M_{m n}(F)$. There is only one such bimodule, and it is isomorphic to the odd part $J_{\overline{1}}$ of the superalgebra $J$ viewed as bimodule over $J_{\overline{0}}$.

Reviewing the irreducible modules above we see that

$$
\Delta(J)=\left\{0 \neq \pm v_{i} \pm v_{j}, 0 \neq \pm w_{k} \pm w_{l}, \pm w_{i} \pm v_{k}\right\} .
$$

\section{Irreducible bimodules over $J P(n), n \geq 2$}

Recall that the Jordan superalgebra $J P(n)$ is the superalgebra of symmetric elements of $M_{n+n}(F)$ with respect to the superinvolution:

$$
\left(\begin{array}{ll}
a & b \\
c & d
\end{array}\right)^{*}=\left(\begin{array}{cc}
d^{t} & -b^{t} \\
c^{t} & a^{t}
\end{array}\right)
$$

So, four examples of unital $J P(n)$-bimodules appear naturally:

(1) the regular bimodule $V=J P(n)$, 
(2) the bimodule $\operatorname{Skew}\left(M_{n+n}(F), *\right)=\left\{\left(\begin{array}{cc}a & h \\ k & -a^{t}\end{array}\right) \mid a \in M_{n}(F), h^{t}=h, k^{t}=\right.$ $-k\}$,

(3)-(4) their opposites.

Lemma 3.1. $J P(n)$ is not isomorphic to $\operatorname{Skew}\left(M_{n+n}(F)\right.$, *) as a $J P(n)$-bimodule.

Proof. Consider the operator

$$
W=R\left(\left(\begin{array}{cc}
0 & 0 \\
e_{11} & 0
\end{array}\right)\right) R\left(\left(\begin{array}{cc}
e_{12} & 0 \\
0 & e_{21}
\end{array}\right)\right) R\left(\left(\begin{array}{cc}
0 & 0 \\
e_{11} & 0
\end{array}\right)\right) U\left(\left(\begin{array}{cc}
e_{12} & 0 \\
0 & e_{21}
\end{array}\right),\left(\begin{array}{cc}
e_{21} & 0 \\
0 & e_{12}
\end{array}\right)\right) .
$$

The straightforward computation shows that $J P(n) W=(0)$, whereas for the element $\left(\begin{array}{cc}0 & e_{11} \\ 0 & 0\end{array}\right) \in \operatorname{Skew}\left(M_{n+n}(F), *\right)$ we have $\left(\begin{array}{cc}0 & e_{11} \\ 0 & 0\end{array}\right) W \neq 0$.

Triangular (super)algebras. Let $L=L_{-}+L_{0}+L_{+}$be a Lie superalgebra, where $L_{-}, L_{0}, L_{+}$are subsuperalgebras of $L, L_{0} \subseteq L_{\overline{0}}$ is abelian, $\left[L_{0}, L_{-}\right] \subseteq L_{-}$, and $\left[L_{0}, L_{+}\right] \subseteq L_{+}$. Suppose that $L_{-}$decomposes into a sum of eigenspaces with respect to the action of $L_{0}, L_{-}=\sum_{\alpha \in \Delta_{-} \subseteq L_{0}^{*}}\left(L_{-}\right)_{\alpha}$ and the semigroup generated by $\Delta_{-}$in $L_{0}^{*}$ does not contain 0 .

Consider a functional $0 \neq \lambda \in L_{0}^{*}$. We say that an $L$-module $V$ is a module of highest weight $\lambda$ if $V$ is generated (as a module) by a nonzero element $v$ such that $L_{+} v=(0)$ and for every $h \in L_{0}, v h=\lambda(h) v$.

For an arbitrary functional $\lambda \in L_{0}^{*}$, there is a unique irreducible module $\operatorname{Irr}(\lambda)$ of the highest weight $\lambda$.

Recall that the TKK Lie algebra $K(J P(n)), n \geq 2$, is the universal central cover $\hat{P}(2 n-1)$ of $P(2 n-1)$. For $n>2$, we have $\hat{P}(2 n-1)=P(2 n-1)$; however, for $n=2$, the superalgebra $\hat{P}(3)$ has a 1 -dimensional center (10 ). Let $H$ be a Cartan subalgebra of $\left[\hat{P}(2 n-1)_{\overline{0}}, \hat{P}(2 n-1)_{\overline{0}}\right]$. Let $\hat{H}=H$ for $n>2$. For $n=2$, let $\hat{H}=H+F z$, where $z$ is a nonzero central element of $\hat{P}(3)$. Then $L=\hat{P}(2 n-1)=$ $\hat{H}+\sum_{\alpha \in \Delta} L_{\alpha}, \Delta=\left\{w_{i}-w_{j},-w_{i}-w_{j}, 1 \leq i \neq j \leq 2 n\right\} \cup\left\{w_{i}+w_{j}, 1 \leq i, j \leq n\right\}$.

Let $V$ be an irreducible unital bimodule over $J=J P(n)$.

The $L$-module $\bar{K}(V)$ decomposes into a direct sum of eigenspaces with respect to $H, \bar{K}(V)=\sum_{\beta} \bar{K}(V)_{\beta}$, with $\beta \in \Delta(J)=\left\{0 \neq \pm w_{i} \pm w_{j}, 1 \leq i, j \leq 2 n\right\}$. Clearly $z \bar{K}(V)_{\beta} \subseteq \bar{K}(V)_{\beta}$ for every $\beta \in \Delta(J)$.

Lemma 3.2. At least one of the subspaces $\bar{K}(V)_{2 w_{i}}, \bar{K}(V)_{w_{i}-w_{j}}$ is not equal to zero.

Proof. Suppose that $\bar{K}(V)_{w_{i}-w_{j}}=\bar{K}(V)_{2 w_{i}}=(0)$ for any $i, j$.

Then,

$$
\bar{K}(V)=\bar{K}(V)_{0}+\sum_{i \neq j} \bar{K}(V)_{w_{i}+w_{j}}+\sum_{i \neq j} \bar{K}(V)_{-w_{i}-w_{j}}+\sum_{i=1}^{2 n} \bar{K}(V)_{-2 w_{i}} .
$$

Choose $v \in \bar{K}(V)_{w_{1}+w_{2}}$.

Consider the elements $q_{-w_{i}-w_{j}}=\left(\begin{array}{cc}0 & e_{i j}-e_{j i} \\ 0 & 0\end{array}\right), q_{w_{i}+w_{j}}=\left(\begin{array}{cc}0 & 0 \\ e_{i j}+e_{j i} & 0\end{array}\right)$, $i \neq j$, from $L$.

We have $v q_{-w_{2}-w_{3}} \in \bar{K}(V)_{-w_{2}-w_{3}+w_{1}+w_{2}}=\bar{K}(V)_{w_{1}-w_{3}}=(0)$. Similarly, $v q_{w_{2}+w_{3}} \in \bar{K}(V)_{w_{2}+w_{3}+w_{1}+w_{2}}=(0)$. Hence $v\left[q_{-w_{2}-w_{3}}, q_{w_{2}+w_{3}}\right]=0$. However, 
$\left[q_{-w_{2}-w_{3}}, q_{w_{2}+w_{3}}\right]=\left(\begin{array}{cc}e_{22}-e_{33} & 0 \\ 0 & -e_{22}+e_{33}\end{array}\right)=h_{w_{2}-w_{3}} \in H$. Therefore $v h_{w_{2}-w_{3}}$ $=\left(w_{2}-w_{3} \mid w_{1}+w_{2}\right) v=v$. We have proved that $\bar{K}(V)_{w_{i}+w_{j}}=(0)$ for any $i, j$.

Similarly, $\bar{K}(V)_{-w_{1}-w_{2}} q_{w_{2}+w_{3}} \subseteq \bar{K}(V)_{-w_{1}+w_{3}}=(0) ; \bar{K}(V)_{-w_{1}-w_{2}} q_{-w_{2}-w_{3}} \subseteq$ $\bar{K}(V)_{-w_{1}-2 w_{2}-w_{3}}=(0)$.

Notice that $-w_{1}-2 w_{2}-w_{3}$ does not lie in $\Delta(J)$ if $n \geq 3$. If $n=2$, then $-w_{1}-2 w_{2}-w_{3}=-w_{2}-\left(w_{1}+w_{2}+w_{3}\right)=-w_{2}-\left(-w_{4}\right)=-w_{2}+w_{4}$ lies in $\Delta(J)$, but $\bar{K}(V)_{-w_{2}+w_{4}}=(0)$ by our assumptions.

For an arbitrary element $v \in \bar{K}(V)_{-w_{1}-w_{2}}$, we have $v h_{w_{2}-w_{3}}=\left(w_{2}-w_{3} \mid\right.$ $\left.-w_{1}-w_{2}\right) v=-v$. Hence $\bar{K}(V)_{-w_{i}-w_{j}}=(0)$, for any $i, j$. Hence, $\bar{K}(V)=$ $\bar{K}(V)_{0}+\sum_{i=1}^{2 n} \bar{K}(V)_{-2 w_{i}}$.

Consider the elements $e_{w_{i}-w_{j}}=\left(\begin{array}{cc}e_{i j} & 0 \\ 0 & e_{j i}\end{array}\right)$. Clearly $\left[e_{w_{i}-w_{j}}, e_{w_{j}-w_{i}}\right]=h_{w_{i}-w_{j}}$.

As above, $\bar{K}(V)_{-2 w_{1}} e_{w_{1}-w_{2}}=\bar{K}(V)_{-2 w_{1}} e_{w_{2}-w_{1}}=(0)$, and therefore $\bar{K}(V)_{-2 w_{1}} h_{w_{1}-w_{2}}=(0)$. As above, this implies that $\bar{K}(V)_{-2 w_{1}}=(0)$. So $\bar{K}(V)=$ $\bar{K}(V)_{0}$, a contradiction that proves the lemma.

Let $\Lambda$ denote the root lattice, $\Lambda=\sum_{i=1}^{2 n} \mathbb{Z} w_{i} / \mathbb{Z}\left(w_{1}+\cdots+w_{2 n}\right)$.

Let $\gamma: \Lambda \rightarrow \mathbb{Z}$ be a functional such that all values $\gamma\left(w_{1}\right), \ldots, \gamma\left(w_{2 n}\right),-\gamma\left(w_{1}\right), \ldots$, $-\gamma\left(w_{2 n}\right)$ are distinct. Let's assume that

$$
\left|\gamma\left(w_{1}\right)\right|>\left|\gamma\left(w_{2}\right)\right|>\max _{3 \leq i \leq 2 n}\left|\gamma\left(w_{i}\right)\right| ; \gamma\left(w_{1}\right)>0, \gamma\left(w_{2}\right)<0 .
$$

We can consider $L_{+}=\sum_{\gamma(\alpha)>0} L_{\alpha}, L_{-}=\sum_{\gamma(\alpha)<0} L_{\alpha}, L=L_{-}+\hat{H}+L_{+}$.

Since all nonzero weights of $\bar{K}(V)$ lie in $\Delta(J)$, it follows that $L_{+} \bar{K}(V)_{2 w_{1}}=(0)$. If $\bar{K}(V)_{2 w_{1}}=(0)$, then $L_{+} \bar{K}(V)_{w_{1}-w_{2}}=(0)$. Both spaces $\bar{K}(V)_{2 w_{1}}$ and $\bar{K}(V)_{w_{1}-w_{2}}$ cannot be simultaneously zero by Lemma 3.2 .

Lemma 3.3. If $n \geq 3$, then the only irreducible finite-dimensional unital Jordan bimodules are the regular bimodule $R$ and the bimodule $S=\operatorname{Skew}\left(M_{n+n}(F), \star\right)$.

Proof. If $n \geq 3$, then $\hat{P}(2 n-1)=P(2 n-1), \hat{H}=H$. Hence if $V$ is a unital finite-dimensional irreducible bimodule over $J P(n)$, the module $\bar{K}(V)$ over $L$ is isomorphic to $\operatorname{Irr}\left(2 w_{1}\right)$ or to $\operatorname{Irr}\left(w_{1}-w_{2}\right)$.

By Lemma 3.2 there are at most two nonisomorphic finite-dimensional irreducible Jordan bimodules over $J P(n)$. By Lemma 3.1 we know the two bimodules $R$ and $S$ are not isomorphic. The lemma is proved.

Now let $n=2$ and let $V$ be a unital irreducible finite dimensional Jordan $J P(2)$ bimodule.

Let

$$
\beta= \begin{cases}2 w_{1} & \text { if } \bar{K}(V)_{2 w_{1}} \neq(0), \\ w_{1}-w_{2} & \text { if } \bar{K}(V)_{2 w_{1}}=(0) .\end{cases}
$$

The eigenspace $\bar{K}(V)_{\beta}$ is invariant with respect to the action of the central element $z$.

Let $W$ be a subspace of $\bar{K}(V)_{\beta}$ such that $z W \subseteq W$. By the Poincaré-BirkhoffWitt theorem the universal enveloping algebra $U(L)$ is $U(L)=U\left(L_{-}\right) U(\hat{H}) U\left(L_{+}\right)$. Hence the $L$-submodule generated by $W$ is $U(L) W=U\left(L_{-}\right) U(\hat{H}) U\left(L_{+}\right) W=$ $U\left(L_{-}\right) W$. Hence $U(L) W \cap \bar{K}(V)_{\beta}=W$. This implies that $z$ acts irreducibly on $\bar{K}(V)_{\beta}$. 
Since $F$ is algebraically closed, it follows that $z$ acts on $\bar{K}(V)_{\beta}$ (and so on $\bar{K}(V)$ ) as a scalar $\alpha \in F$.

Definition 3.4. We say that a unital bimodule $V$ over $J P(2)$ is of level $\alpha \in F$ if $z$ acts as $\alpha$ on $\bar{K}(V)$.

From what we proved above it follows that for an arbitrary element $\alpha \in F$ there are at most two nonisomorphic unital irreducible finite-dimensional Jordan bimodules over $J P(2)$ of level $\alpha$.

We will now describe the explicit realizations of two non-isomorphic $J P(2)$ bimodules of level $\alpha$.

Consider the associative commutative algebra $\Phi=F 1+F t, t^{2}=0$, and its derivation $d: \Phi \rightarrow \Phi$ such that $d(t)=\alpha t$.

Let $W$ be the Weyl algebra of the differential algebra $(\Phi, d)$,

$$
W=\sum_{i \geq 0} \Phi d^{i}=F[d]+t F[d]
$$

$d t-t d=d(t)=\alpha t$.

For an arbitrary $k \geq 0$ the subspace $t d^{k} F[d]$ is an ideal of $W$.

The following embedding of $J P(2)$ into $M_{2+2}(W)^{(+)}$was described in 8 . The Jordan superalgebra of $M_{2+2}(W)^{(+)}$,

$$
J=\left\{\left(\begin{array}{cc}
a & 0 \\
h & a^{t}
\end{array}\right) \mid a, h \in M_{2}(F), h^{t}=h\right\}+F\left(\begin{array}{cccc}
0 & 0 & 0 & -1 \\
0 & 0 & 1 & 0 \\
0 & d & 0 & 0 \\
-d & 0 & 0 & 0
\end{array}\right),
$$

is isomorphic to $J P(2)$.

Denote $x=\left(\begin{array}{cccc}0 & 0 & 0 & -1 \\ 0 & 0 & 1 & 0 \\ 0 & d & 0 & 0 \\ -d & 0 & 0 & 0\end{array}\right)$. The central element $z$ in $K(J)$ is $z=\left[x^{-}, x^{+}\right]$.

The inner derivation $R(x)^{2}$ acts on $M_{2+2}(t F[d])$ as multiplication by $\alpha$. Hence $M_{2+2}(t F[d])$ is a bimodule over $J$ of level $\alpha$.

Let $e=\left(\begin{array}{cccc}t & 0 & 0 & 0 \\ 0 & t & 0 & 0 \\ 0 & 0 & t & 0 \\ 0 & 0 & 0 & t\end{array}\right)$. Then we have

Lemma 3.5. $e \cdot J$ is an irreducible $J$-subbimodule of $M_{2+2}(t F[d])$.

Proof. First notice that $\left[e,\left(\begin{array}{cc}a & 0 \\ h & a^{t}\end{array}\right)\right]=0$, which implies that $D\left(e,\left(\begin{array}{cc}a & 0 \\ h & a^{t}\end{array}\right)\right)=0$. Hence we need only to check that $(e \cdot J) \cdot x \subseteq e \cdot J$.

Now $e R(x)^{2}=\alpha e$. That's why we need to consider only $\left(e \cdot\left(\begin{array}{cc}a & 0 \\ h & a^{t}\end{array}\right)\right) x$. As above, $[e, x]=\alpha\left(\begin{array}{cc}0 & 0 \\ t k & 0\end{array}\right)$, where $k=\left(\begin{array}{cc}0 & 1 \\ -1 & 0\end{array}\right)$. Now,

$$
\left[\left(\begin{array}{cc}
a & 0 \\
0 & a^{t}
\end{array}\right),\left(\begin{array}{cc}
0 & 0 \\
t k & 0
\end{array}\right)\right]=t\left[\left(\begin{array}{cc}
a & 0 \\
0 & a^{t}
\end{array}\right),\left(\begin{array}{cc}
0 & 0 \\
k & 0
\end{array}\right)\right]=t\left(\begin{array}{cc}
0 & 0 \\
a^{t} k-k a & 0
\end{array}\right)
$$


$a^{t} k-k a \in H$. Commuting $\left(\begin{array}{ll}0 & 0 \\ h & 0\end{array}\right)$ with $\left(\begin{array}{ll}0 & 0 \\ k & 0\end{array}\right)$ we get 0 . This proves that $(e . J) . J \subseteq e . J$.

The even part of this bimodule $e . J_{\overline{0}}$ is the regular irreducible bimodule over $J_{\overline{0}}=M_{2}(F)^{(+)}$. Hence, if $e J$ has a proper subbimodule, then it has to be contained in the odd part and, thus, be killed by $J_{\overline{1}}$. Let $v \in e J_{\overline{1}}$ be an element killed by $J_{\overline{1}}$. Let $e_{1}=\left(\begin{array}{cc}e_{11} & 0 \\ 0 & e_{11}\end{array}\right), e_{2}=\left(\begin{array}{cc}e_{22} & 0 \\ 0 & e_{22}\end{array}\right)$ be the orthogonal idempotents from $J_{\overline{0}}$.

We have $J D\left(v,\left[J_{\overline{1}}, J_{\overline{1}}\right] \subseteq J D\left(v J_{\overline{1}}, J_{\overline{1}}\right)=(0)\right.$. In particular $e_{1} D\left(v, e_{1}-e_{2}\right)=0$, which implies that $v$ lies in the Peirce component $\left\{e_{1}, e J_{\overline{1}}, e_{1}\right\}+\left\{e_{2}, J_{\overline{1}}, e_{2}\right\}$. Hence, $v=e\left(\begin{array}{ll}0 & 0 \\ h & 0\end{array}\right), h \in H(2)$. Now $\left(e\left(\begin{array}{ll}0 & 0 \\ h & 0\end{array}\right)\right) x=0$ implies $h=0$. The lemma is proved.

Definition 3.6. We will denote this bimodule as $R(\alpha)$. It is easy to see that $R(0)$ is the regular bimodule.

Consider the $J$-bimodule $V=M_{2+2}(t F[d] / t d F[d]), \operatorname{dim}_{F} V=16$. We will identify $R(\alpha)$ with the subbimodule of $V$ generated by $\bar{e}=e+M_{2+2}(t d F[d])$.

Lemma 3.7. The J-bimodule $S(\alpha)=V / R(\alpha)$ is irreducible.

Proof. Consider a composition series of $V / R(\alpha)$ with irreducible factors. Suppose that it has more than one factor. Then all these factors have dimensions $<8$. We have shown above that for each $\alpha, J$ has at most two irreducible finite-dimensional unital Jordan bimodules of level $\alpha$. One of these irreducible bimodules is $R(\alpha)$. Let $V^{\prime}$ be another irreducibe $J$-bimodule of level $\alpha, \operatorname{dim}_{F} V^{\prime}<8$. Let $q$ be the length of the composition series of $V / R(\alpha)$.

Notice that $D\left(J_{\overline{0}}, J_{\overline{0}}\right) \simeq s l_{2}$ and that

$$
\operatorname{dim}_{F}\left\{v \in V / R(\alpha) \mid D\left(J_{\overline{0}}, J_{\overline{0}}\right) v=(0)\right\}=q \cdot \operatorname{dim}_{F}\left\{v \in V^{\prime} \mid D\left(J_{\overline{0}}, J_{\overline{0}}\right) v=(0)\right\} .
$$

However,

$$
\left\{v \in V \mid D\left(J_{\overline{0}}, J_{\overline{0}}\right) v=(0)\right\}=\left\{t\left(\begin{array}{cccc}
\xi & 0 & 0 & 0 \\
0 & \xi & 0 & 0 \\
0 & 0 & \eta & 0 \\
0 & 0 & 0 & \eta
\end{array}\right)\right\},
$$

hence $\operatorname{dim}\left\{v \in V / R(\alpha) \mid D\left(J_{\overline{0}}, J_{\overline{0}}\right) v=(0)\right\}=1$. This proves the lemma.

Lemma 3.8. $R(\alpha) \neq S(\alpha)$.

Proof. The modules $\bar{K}(R(\alpha))$ and $\bar{K}(S(\alpha))$ over $\hat{P}(3)$ have different sets of weights.

Remark 3.9. If $\alpha \neq 0$, then the bimodule $V$ is indecomposable ([13]).

We have proved the following theorem.

Theorem 3.10. The only finite-dimensional unital irreducible Jordan bimodules over $J P(2)$ are $R(\alpha), R(\alpha)^{o p}, S(\alpha), S(\alpha)^{o p}, \alpha \in F$. 
4. Irreducible bimodules over $M_{m+n}(F)^{(+)}, m \geq n, n+m \geq 3$

We have already mentioned in Section 3 that the only irreducible unital Jordan bimodules over $M_{n}(F)^{+}, n \geq 2$, are the regular bimodule and $\left(\begin{array}{cc}0 & H(n) \\ 0 & 0\end{array}\right)$, $\left(\begin{array}{cc}0 & 0 \\ H(n) & 0\end{array}\right),\left(\begin{array}{cc}0 & K(n) \\ 0 & 0\end{array}\right),\left(\begin{array}{cc}0 & 0 \\ K(n) & 0\end{array}\right)$.

The corresponding irreducible modules over the TKK Lie algebra

$$
s l(2 n)=K\left(M_{n}(F)^{(+)}\right)
$$

are the regular module and $\left(\begin{array}{cc}0 & H(2 n) \\ 0 & 0\end{array}\right),\left(\begin{array}{cc}0 & 0 \\ H(2 n) & 0\end{array}\right),\left(\begin{array}{cc}0 & K(2 n) \\ 0 & 0\end{array}\right),\left(\begin{array}{cc}0 & 0 \\ K(2 n) & 0\end{array}\right)$. For $n \geq 3$ all these modules are not isomorphic. For $n=2,\left(\begin{array}{cc}0 & K(n) \\ 0 & 0\end{array}\right) \simeq$ $\left(\begin{array}{cc}0 & 0 \\ K(n) & 0\end{array}\right)$. Hence, if $n \geq 3$, there are 5 nonisomorphic irreducible unital bimodules over $M_{n}(F)^{(+)}$. Over $M_{2}(F)^{(+)}$there are only 4 .

If one of the numbers $m, n$ is even, then $M_{m+n}(F)$ is equipped with a superinvolution. Hence, we can repeat the construction above and get 5 non-isomorphic unital irreducible Jordan bimodules over $M_{m+n}(F)^{(+)}$.

Definition 4.1. Let $A=A_{\overline{0}}+A_{\overline{1}}$ be an associative superalgebra. A graded mapping $\star: A \rightarrow A, A_{\bar{i}}^{\star}=A_{\bar{i}}$, is called a pseudoinvolution if $(a b)^{\star}=(-1)^{|a||b|} b^{\star} a^{\star}$, $a^{\star \star}=(-1)^{|a|} a$, for any $a, b \in A_{\overline{0}} \cup A_{\overline{1}}$.

Example 4.2. For $A=M_{m+n}(F),\left(\begin{array}{ll}a & b \\ c & d\end{array}\right)^{\star}=\left(\begin{array}{cc}a^{t} & -c^{t} \\ b^{t} & d^{t}\end{array}\right)$ is a pseudoinvolution.

Let $\star: A \rightarrow A$ be a pseudoinvolution. Then $A^{(+)} \rightarrow M_{2}(A)^{(+)}$is still an embedding of Jordan superalgebras. Let $W$ be a subspace of $A$ such that for arbitrary elements $w \in W, a \in A$,

$$
a w+(-1)^{|a||w|} w a^{\star} \in W .
$$

Then $W^{u p}=\left(\begin{array}{cc}0 & W \\ 0 & 0\end{array}\right)$ is a Jordan bimodule over $\left\{\left(\begin{array}{cc}a & 0 \\ 0 & a^{\star}\end{array}\right), a \in A\right\}$.

Similarly, if $W$ is a subspace of $A$ and for arbitrary elements $w \in W, a \in A$, we have

$$
a^{\star} w+(-1)^{|a||w|} w a \in W .
$$

Then $W^{\text {down }}=\left(\begin{array}{cc}0 & 0 \\ W & 0\end{array}\right)$ is a Jordan bimodule over $\left\{\left(\begin{array}{cc}a & 0 \\ 0 & a^{\star}\end{array}\right) \mid a \in A\right\} \simeq A^{(+)}$.

Notice that the conditions, $(\mathrm{C} 1)$ and $(\mathrm{C} 2)$ are different.

Now we will give some examples of such subspaces. Let $W_{1}=\left\{\left(\begin{array}{cc}K(m) & b \\ -b^{t} & H(n)\end{array}\right)\right.$, $\left.b \in M_{m, n}(F)\right\}$. A straightforward computation shows that it satisfies (C1). Similarly, $W_{2}=\left\{\left(\begin{array}{cc}H(m) & b \\ b^{t} & K(n)\end{array}\right), b \in M_{m, n}(F)\right\}$ satisfies (C1). The subspaces $W_{3}=$ $\left\{\left(\begin{array}{cc}H(m) & b \\ -b^{t} & K(n)\end{array}\right), b \in M_{m, n}(F)\right\}$ and $W_{4}=\left\{\left(\begin{array}{cc}K(m) & b \\ b^{t} & H(n)\end{array}\right), b \in M_{m, n}(F)\right\}$ satisfy $(\mathrm{C} 2)$. 
So, together with the regular bimodule we have 5 irreducible unital bimodules (plus the 5 opposite) over $M_{m+n}(F)^{(+)}$(they are not isomorphic).

Now let $V$ be an irreducible unital Jordan bimodule, $J=M_{m+n}(F)^{(+)}, L=$ $K(J)=\left\{\left(\begin{array}{ll}a & b \\ c & d\end{array}\right)\right\}, a \in M_{2 m}(F), d \in M_{2 n}(F), \operatorname{tr}(a)=\operatorname{tr}(d)$.

Then

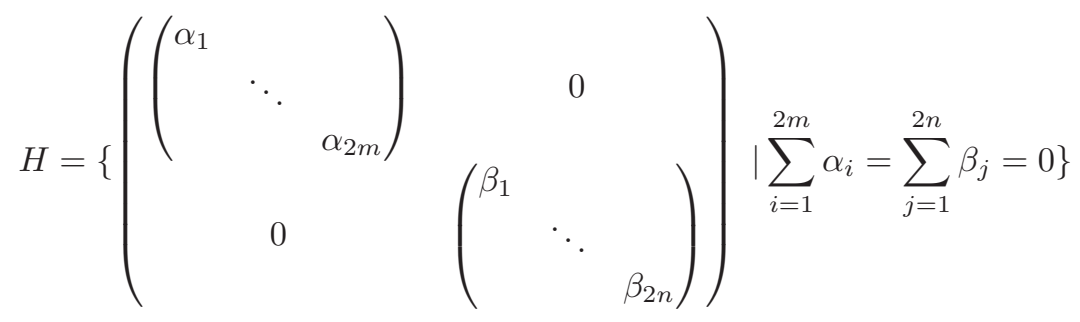

is a Cartan subalgebra of $\left[L_{\overline{0}}, L_{\overline{0}}\right]$,

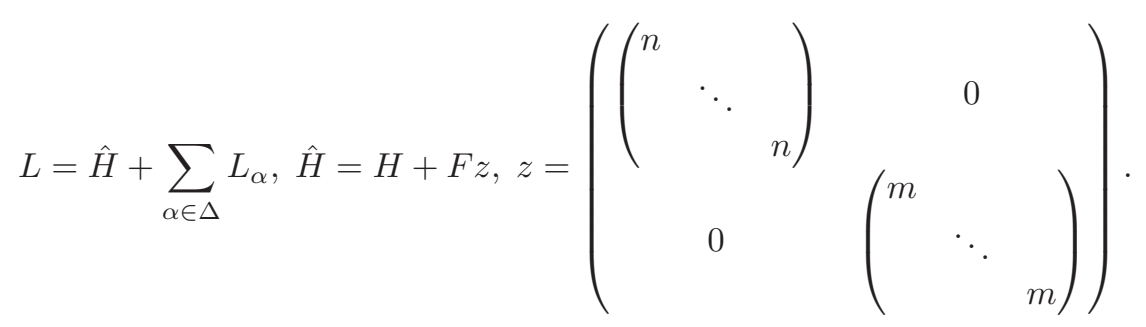

As shown in Section $3, \Delta(J)=\left\{w_{i}-w_{j}, 1 \leq i \neq j \leq 2 n, v_{p}-v_{q}, 1 \leq p \neq q \leq\right.$ $\left.2 m, w_{i}-v_{p}, v_{p}-w_{i}\right\}$. Let $\Delta(V)$ denote the set of nonzero weights of the irreducible $L$-module $\bar{K}(V)$ with respect to $H$. Then, $\Delta(V) \subseteq \Delta(J)$.

Let $\gamma: \Lambda \rightarrow R$ be a functional such that $\left|\gamma\left(w_{1}\right)\right|>\left|\gamma\left(w_{2}\right)\right|>\left|\gamma\left(w_{3}\right)\right|>\left|\gamma\left(w_{4}\right)\right| \geq$ $\max \left\{\left|\gamma\left(w_{i}\right)\right|,\left|\gamma\left(v_{p}\right)\right|, i \geq 4,1 \leq p \leq 2 m\right\}$ and $\gamma\left(w_{1}\right)>0, \gamma\left(w_{2}\right)<0, \gamma\left(w_{4}\right)<0$, $-\gamma\left(w_{2}\right)>\frac{1}{2}\left(\gamma\left(w_{1}\right)+\gamma\left(w_{3}\right)\right)$.

Notice that such functional exists, because $n \geq 2$.

We have

$$
L=L_{-}+\hat{H}+L_{+}, \text {where } L_{-}=\sum_{\gamma(\lambda)<0} L_{\lambda}, L_{+}=\sum_{\gamma(\lambda)>0} L_{\lambda} .
$$

Let $\bar{K}(V)_{\beta} \neq(0)$ and $\gamma(\beta)=\max \gamma(\Delta(V))$. Then, as we have seen before, $z$ (central element) acts irreducibly on $\bar{K}(V)_{\beta}$, which implies that $z$ acts as a scalar $\alpha$ on $\bar{K}(V)$. We will refer to this $\alpha$ as the level of the module $V$.

The module $\bar{K}(V)$ is uniquely determined (up to isomorphism) by $\beta$ and by the level.

Let's denote $q_{w_{i}-v_{p}}=\left(\begin{array}{cc}0 & e_{i p} \\ 0 & 0\end{array}\right)$, for $1 \leq i \leq 2 m, 1 \leq p \leq 2 n$, and $q_{v_{p}-w_{i}}=$ $\left(\begin{array}{cc}0 & 0 \\ e_{i p} & 0\end{array}\right)$. Then $\left[q_{w_{i}-v_{j}}, q_{v_{j}-w_{i}}\right]=\left(\begin{array}{cc}e_{i i} & 0 \\ 0 & e_{p p}\end{array}\right)=h_{w_{i}+v_{p}}$.

Lemma 4.3. If $W$ is a root graded module over $L$ and $W_{ \pm w_{i} \pm w_{j}}=(0)$, for arbitrary $1 \leq i, j \leq 2 m$, then $W L=(0)$.

Proof. The weight space $W_{ \pm w_{1} \pm v_{1}}$ is killed both by $q_{w_{2}-v_{1}}$ and by $q_{-w_{2}+v_{1}}$; hence it is killed by $h_{w_{2}+v_{1}}=\left[q_{w_{2}-v_{1}}, q_{-w_{2}+v_{1}}\right]$. Similarly, $V_{ \pm w_{1} \pm v_{1}}$ is killed by $q_{w_{2}-v_{2}}$ and by $q_{-w_{2}+v_{2}}$; hence it is killed by $h_{w_{2}+v_{2}}=\left[q_{w_{2}-v_{2}}, q_{-w_{2}+v_{2}}\right]$. 
Now $h_{v_{1}-v_{2}}=h_{w_{2}+v_{1}}-h_{w_{2}+v_{2}}$ kills $W_{ \pm w_{1} \pm v_{1}}$, but $\left\langle v_{1}-v_{2} \mid \pm w_{1} \pm v_{1}\right\rangle= \pm 1$. Hence $W_{ \pm w_{1} \pm v_{1}}=(0)$.

Hence, $W=\sum W_{ \pm v_{p} \pm v_{q}}$ and hence $L_{\overline{1}} W=(0)$, which implies $L W=(0)$. The lemma is proved.

Lemma 4.4. There are 5 possibilities for the highest weight $\lambda$ : $2 w_{1}, w_{1}-w_{2}$, $-2 w_{2}, w_{1}+w_{3},-w_{2}-w_{4}$.

Proof. If $\bar{K}(V)_{2 w_{1}} \neq(0)$, then $2 w_{1}$ is clearly the highest weight.

If $\bar{K}(V)_{2 w_{1}}=(0)$, then, since all $\bar{K}(V)_{2 w_{i}}$ are conjugate via the Weyl group, then $\bar{K}(V)_{2 w_{i}}=(0)$ for an arbitrary $1 \leq i \leq 2 m$. If $\bar{K}(V)_{w_{1}-w_{2}} \neq(0)$, then $w_{1}-w_{2}$ is the highest weight.

If $\bar{K}(V)_{w_{1}-w_{2}}=(0)$, then $\bar{K}(V)_{w_{i}-w_{j}}=(0)$ for an arbitrary $1 \leq i \neq j \leq 2 m$. If $\bar{K}(V)_{2 w_{1}}=(0), \bar{K}(V)_{w_{1}-w_{2}}=(0)$ and $\bar{K}(V)_{-2 w_{2}} \neq(0)$, then $-2 w_{2}$ is the highest weight. In case that it is zero, then, as above, $\bar{K}(V)_{-2 w_{i}}=(0)$ for an arbitrary $1 \leq i \leq 2 m$. Similarly, if $\bar{K}(V)_{2 w_{1}}=(0), \bar{K}(V)_{w_{1}-w_{2}}=(0), \bar{K}(V)_{-2 w_{1}}=(0)$ and $(0) \neq \bar{K}(V)_{w_{1}+w_{3}}$, then $w_{1}+w_{3}$ is the highest weight.

Finally, if $\bar{K}(V)_{2 w_{1}}=(0), \bar{K}(V)_{w_{1}-w_{2}}=(0), \bar{K}(V)_{-2 w_{1}}=(0),(0)=\bar{K}(V)_{w_{1}+w_{3}}$ and $\bar{K}(V)_{-w_{2}-w_{4}} \neq(0)$, then the highest weight is $-w_{2}-w_{4}$. If $\bar{K}(V)_{\beta}=(0)$, for $\beta=2 w_{1}, w_{1}-w_{2},-2 w_{2}, w_{1}+w_{2},-w_{2}-w_{4}$, then by Lemma $4.3 L \bar{K}(V)=(0)$, a contradiction. The lemma is proved.

Remark 4.5. If $m=2$, then $w_{1}+w_{3}=-w_{2}-w_{4}$.

It is easy to see that $\hat{H}=H+F z=H+F h_{w_{i}+v_{p}}$, for arbitrary $i, p$.

Lemma 4.6. In the cases $\lambda=2 w_{1}, w_{1}-w_{2},-2 w_{3}$ in Lemma 4.4 the action of $\hat{H}$ on $\bar{K}(V)$ is uniquely determined. If $n \geq 3$, then in the cases $\lambda=w_{1}+w_{3},-w_{2}-w_{4}$ the action of $\hat{H}$ is also uniquely determined. If $n=2$ and $\lambda=w_{1}+w_{3}=-w_{2}-w_{4}$, then there are two ways in which $\hat{H}$ can act on $\bar{K}(V)$.

Proof. Suppose that $\lambda=2 w_{1}, \bar{K}(V)_{2 w_{1}} \neq(0)$. We have $\bar{K}(V)_{2 w_{1}} q_{w_{3}-v_{1}}=(0)$ and $\bar{K}(V)_{2 w_{1}} q_{v_{1}-w_{3}}=(0)$. Hence $\bar{K}(V)_{2 w_{1}} h_{w_{3}+v_{1}}=(0)$ which determines the action of $\hat{H}$ on $\bar{K}(V)$.

Now suppose that $\bar{K}(V)_{2 w_{i}}=(0)$ for $1 \leq i \leq 2 m, \lambda=w_{1}-w_{2}$ and $\bar{K}(V)_{w_{1}-w_{2}} \neq$ (0). Again $\bar{K}(V)_{w_{1}-w_{2}} q_{w_{3}-v_{1}}=\bar{K}(V)_{w_{1}-w_{2}} q_{v_{1}-w_{3}}=(0)$. Hence $\bar{K}(V)_{w_{1}-w_{2}} h_{w_{3}+v_{1}}$ $=(0)$, which determines uniquely the action of $\hat{H}$ on $\bar{K}(V)$.

Let $\bar{K}(V)_{2 w_{i}}=\bar{K}(V)_{w_{i}-w_{j}}=(0)$, for arbitrary $1 \leq i \neq j \leq 2 m, \lambda=-2 w_{2}$, $\bar{K}(V)_{-2 w_{2}} \neq(0)$. As above, $\bar{K}(V)_{-2 w_{2}} q_{w_{3}-v_{1}}=\bar{K}(V)_{-2 w_{2}} q_{v_{1}-w_{3}}=(0)$; hence $\bar{K}(V)_{-2 w_{2}} h_{w_{3}+v_{1}}=(0)$ and the action of $\hat{H}$ is uniquely determined.

Let $m \geq 3$ and $\lambda=w_{1}+w_{3}$. Then

$$
\bar{K}(V)_{w_{1}+w_{3}} q_{w_{2}-v_{1}}=\bar{K}(V)_{w_{1}+w_{3}} q_{v_{1}-w_{2}}=(0),
$$

which determines the action of $\hat{H}$ over $\bar{K}(V)$.

With the same assumptions, let $\lambda=-w_{2}-w_{4}$. Then

$$
\bar{K}(V)_{-w_{2}-w_{4}} q_{w_{3}-v_{1}}=\bar{K}(V)_{-w_{2}-w_{4}} q_{v_{1}-w_{3}}=(0),
$$

and the action of $\hat{H}$ is determined by the fact that $\bar{K}(V)_{-w_{2}-w_{4}} h_{w_{3}+v_{1}}=(0)$.

Now suppose that $m=2, \lambda=w_{1}+w_{3}=-w_{2}-w_{4}$. 
Case 1. $\bar{K}(V)_{w_{1}+v_{1}}=(0)$.

In this case, $\bar{K}(V)_{w_{1}+w_{3}} q_{-w_{3}+v_{1}}=\bar{K}(V)_{w_{1}+w_{3}} q_{w_{3}-v_{1}}=(0)$. Hence the action of $\hat{H}$ is determined since $\bar{K}(V)_{w_{1}+w_{3}} h_{w_{3}+v_{1}}=(0)$.

Case 2. $\bar{K}(V)_{w_{1}+v_{1}} \neq(0)$.

In this case, $\bar{K}(V)_{w_{1}+v_{1}}$ generates $\bar{K}(V)$. The action of $\hat{H}$ on $\bar{K}(V)$ is determined by its action on $\bar{K}(V)_{w_{1}+v_{1}}$. We have

$$
\bar{K}(V)_{w_{1}+v_{1}} q_{w_{2}-v_{2}}=\bar{K}(V)_{w_{1}+v_{1}} q_{v_{2}-w_{2}}=(0),
$$

so the action of $\hat{H}$ on $\bar{K}(V)_{w_{1}+v_{1}}$ is determined. The lemma is proved.

From Lemma 4.6 it follows that there are at most 5 (up to opposites) unital irreducible Jordan bimodules over $M_{m+n}(F)^{(+)}$. Since we have described 5 such bimodules at the beginning of this section, it implies the following theorem.

Theorem 4.7. The only finite-dimensional unital irreducible Jordan bimodules over $M_{m+n}(F)^{(+)}, m+n \geq 3$, are the regular bimodule, $W_{1}^{u p}, W_{2}^{u p}, W_{3}^{\text {down }}, W_{4}^{\text {down }}$ and their opposites.

\section{Unital irreducible Bimodules over $M_{1+1}(F)^{(+)}$}

In 7 unital bimodules over $M_{1+1}(F)$ were studied. Here a new approach, that is included for completeness, is given.

Let $\{e, f, x, y\}$ be the standard base of $J=M_{1+1}(F)^{(+)}$; that is, $e=\left(\begin{array}{ll}1 & 0 \\ 0 & 0\end{array}\right)$, $f=\left(\begin{array}{ll}0 & 0 \\ 0 & 1\end{array}\right), x=\left(\begin{array}{ll}0 & 1 \\ 0 & 0\end{array}\right), y=\left(\begin{array}{ll}0 & 0 \\ 1 & 0\end{array}\right)$. Thus $J_{\overline{0}}=F e+F f, J_{\overline{1}}=F x+F y$, $[x, y]=e-f$.

For arbitrary scalars $\alpha, \beta, \gamma \in F$ define a 4-dimensional $J$-bimodule, denoted $V(\alpha, \beta, \gamma)$, with a base $v, w \in V_{\overline{0}}, z, t \in V_{\overline{1}}$ and the multiplication table

$$
\begin{gathered}
v e=v, w e=0, z e=\frac{1}{2} z, t e=\frac{1}{2} t, \\
v f=0, w f=w, z f=\frac{1}{2} z, t f=\frac{1}{2} t, \\
v x=z, w x=(\gamma-1) z-2 \alpha t, z x=\alpha v, t x=\frac{1}{2}((\gamma-1) v-w), \\
v y=t, w y=2 \beta z-(\gamma+1) t, z y=\frac{1}{2}(\gamma+1) v+\frac{1}{2} w, t y=\beta v .
\end{gathered}
$$

Remark 5.1. The operators $R(x)^{2}, R(y)^{2}, R(x) R(y)=R(y) R(x)$ act on $V(\alpha, \beta, \gamma)$ as $\alpha, \beta, \gamma$, respectively.

Lemma 5.2. $V(\alpha, \beta, \gamma)$ is a Jordan bimodule over $J$.

Proof. Choosing an appropriate base in $J_{\overline{1}}$ we will assume that $\gamma=0$. Let us embed $M_{1+1}(F)^{(+)}$into $M_{2+2}(F)^{(+)}$via

$$
e \equiv\left(\begin{array}{cc}
I & 0 \\
0 & 0
\end{array}\right), f \equiv\left(\begin{array}{cc}
0 & 0 \\
0 & I
\end{array}\right), x \equiv\left(\begin{array}{cc}
0 & I \\
A & 0
\end{array}\right), y \equiv\left(\begin{array}{cc}
0 & B \\
I & 0
\end{array}\right)
$$

where $A=\left(\begin{array}{cc}0 & 0 \\ 0 & 2 \alpha\end{array}\right), B=\left(\begin{array}{cc}2 \beta & 0 \\ 0 & 0\end{array}\right), I=\left(\begin{array}{ll}1 & 0 \\ 0 & 1\end{array}\right)$. 
Let $v=\left(\begin{array}{cc}E & 0 \\ 0 & 0\end{array}\right), E=\left(\begin{array}{ll}0 & 1 \\ 0 & 0\end{array}\right)$. Then the $J$-submodule generated by the element $v$ is $V(\alpha, \beta, 0)$. The lemma is proved.

Remark 5.3. It is easy to see that $v=\{e, v, e\}, w=v U(x, y), z=v x, t=v y$.

Lemma 5.4. If $\gamma^{2}-4 \alpha \beta-1 \neq 0$, then the bimodule $V(\alpha, \beta, \gamma)$ is irreducible. If $\gamma^{2}-4 \alpha \beta-1=0$, then $F w+F w x$ is the only proper subbimodule of $V(\alpha, \beta, \gamma)$.

Proof. Let $V^{\prime}$ be a nonzero subbimodule of $V(\alpha, \beta, \gamma)$. First we will notice that $V^{\prime} \cap V_{\overline{0}} \neq(0)$. Indeed, otherwise $V^{\prime} x=V^{\prime} y=(0)$. We apply the Jordan identity $V^{\prime}(R(x) R(e) R(y)-R(y) R(e) R(x)-R([x, y] e)-R(x e) R(y)+R(y e) R(x)-$ $R([x, y]) R(e))=(0)$ and we get $V^{\prime}=(0)$. If $\left\{e, V^{\prime}, e\right\} \neq(0)$, then $v \in V^{\prime}$ and therefore $V^{\prime}=V$. If $\left\{e, V^{\prime}, e\right\}=(0)$, then $V_{0}^{\prime}=F w$. We have $w U(x, y)=$ $v U(x, y)^{2}=\left(\gamma^{2}-1-4 \alpha \beta\right) v m$. Hence, if $\gamma^{2}-1-4 \alpha \beta \neq 0$ we still get $V^{\prime}=V$. The lemma is proved.

For $\alpha, \beta, \gamma \in F$ such that $\gamma^{2}-1-4 \alpha \beta=0$, denote $V^{(f)}(\alpha, \beta, \gamma)=F w+w J_{\overline{1}}$, $V^{(e)}(\alpha, \beta, \gamma)=V(\alpha, \beta, \gamma) / V^{(f)}(\alpha, \beta, \gamma)$.

Theorem 5.5. Every irreducible finite-dimensional unital Jordan J-bimodule is isomorphic to one of $V(\alpha, \beta, \gamma), \gamma^{2}-1-4 \alpha \beta \neq 0$ or $V^{(f)}(\alpha, \beta, \gamma)$ or $V^{(e)}(\alpha, \beta, \gamma)$, if $\gamma^{2}-1-4 \alpha \beta=0$ or its opposite.

Proof. Let $V$ be an irreducible finite-dimensional Jordan $J$-bimodule. Up to passing to an opposite, we can assume that $V$ is in the normal form, $V_{\overline{0}}=\left\{e, V_{\overline{0}}, e\right\}+$ $\left\{f, V_{\overline{0}}, f\right\}, V_{\overline{1}}=\left\{e, V_{\overline{1}}, f\right\}$.

The operators $R(x)^{2}, R(y)^{2}, R(x) R(y)+R(y) R(x)$ commute with the action of $J$; hence by Schur's Lemma they act as scalars $\alpha, \beta, \gamma$, respectively.

Let $W$ be a subspace of $\left\{e, V_{\overline{0}}, e\right\}$. We claim that $U=W+W U\left(J_{\overline{1}}, J_{\overline{1}}\right)+W J_{\overline{1}}$ is a $J$-bimodule.

Indeed, $W J_{\overline{1}} \subseteq\left\{e, V_{\overline{1}}, f\right\}, W U\left(J_{\overline{1}}, J_{\overline{1}}\right) \subseteq\left\{f, V_{\overline{0}}, f\right\}$. Hence each summand $W, W U\left(J_{\overline{1}}, J_{\overline{1}}\right), W J_{\overline{1}}$ is invariant under the multiplication by $J_{\overline{0}}$. Furthermore, $R\left(J_{\overline{1}}\right) R\left(J_{\overline{1}}\right) \subseteq U\left(J_{\overline{1}}, J_{\overline{1}}\right)+D\left(J_{\overline{1}}, J_{\overline{1}}\right)+R\left(J_{\overline{0}}\right)$. Hence $W R\left(J_{\overline{1}}\right) R\left(J_{\overline{1}}\right) \subseteq U$ and, more generally, $W R(J) R(J) \subseteq U$.

It remains to show that $W U\left(J_{\overline{1}}, J_{\overline{1}}\right) R\left(J_{\overline{1}}\right) \subseteq W J_{\overline{1}}$. We have $U\left(J_{\overline{1}}, J_{\overline{1}}\right) \subseteq$ $R\left(J_{\overline{1}}\right) R\left(J_{\overline{1}}\right)+R\left(J_{\overline{0}}\right)$.

By the identity 4 (see the Introduction),

$$
R\left(J_{\overline{1}}\right) R\left(J_{\overline{1}}\right) R\left(J_{\overline{1}}\right) \subseteq R(J) R(J)+D\left(J_{\overline{1}}, J_{\overline{1}}\right) R\left(J_{\overline{1}}\right) .
$$

Since $D\left(J_{\overline{1}}, J_{\overline{1}}\right)$ acts as multiplication by scalars, the claim follows.

Similarly, if $W \subseteq\left\{f, V_{\overline{0}}, f\right\}$, then $W+W U\left(J_{\overline{1}}, J_{\overline{1}}\right)+W J_{\overline{1}}$ is a $J$-bimodule. Since $V$ is irreducible it follows that $\operatorname{dim}_{F}\left\{e, V_{\overline{0}}, e\right\} \leq 1, \operatorname{dim}_{F}\left\{f, V_{\overline{0}}, f\right\} \leq 1, \operatorname{dim}_{F} V_{\overline{1}} \leq 2$.

Suppose that $1+4 \alpha \beta-\gamma^{2} \neq 0$. We will show that $V \simeq V(\alpha, \beta, \gamma)$. As we have seen above, $V_{\overline{0}} \neq(0)$. The operator $U(x, y)^{2}$ acts on $V_{\overline{0}}$ as multiplications by $\gamma^{2}-4 \alpha \beta-1$. Hence both $\left\{e, V_{\overline{0}}, e\right\}$ and $\left\{f, V_{\overline{0}}, f\right\}$ are different from zero. Choose $0 \neq v \in\left\{e, V_{\overline{0}}, e\right\}$. Let $w=v U(x, y) \in\left\{f, V_{\overline{0}}, f\right\}$.

We claim that the elements $v x$ and $v y$ are linearly independent. Indeed, suppose that $v y=\xi v x, \xi \in F$. Then $v R(y) R(x)=\xi \alpha v$ and

$$
\begin{aligned}
v U(x, y) & =v(R(x) R(y)-R(y) R(x)-R(e-f)) \\
& =v(R(x) R(y)+R(y) R(x)-2 R(y) R(x)-R(e-f)) \in F v,
\end{aligned}
$$


a contradiction. Hence $v, w, v x, v y$ is a base of $V$ with the multiplication table as in $V(\alpha, \beta, \gamma)$.

Now let $1+4 \alpha \beta-\gamma^{2}=0$. Then $V_{\overline{0}} U(x, y)^{2}=(0)$. Suppose that $\left\{e, V_{\overline{0}}, e\right\} \neq(0)$, $0 \neq v \in\left\{e, V_{\overline{0}}, e\right\}$. If $w=v U(x, y) \neq 0$, then $V$ is generated by $w, w U(x, y), w x, w y$; but $w U(x, y)=0$, which implies $\operatorname{dim}_{F} V_{\overline{0}} \leq 1$, a contradiction. Hence $v U(x, y)=0$. In this case $V \simeq V^{(e)}(\alpha, \beta, \gamma)$. If $\left\{e, V_{\overline{0}}, e\right\}=(0),\left\{f, V_{\overline{0}}, f\right\} \neq(0)$, then $V \simeq$ $V^{(f)}(\alpha, \beta, \gamma)$. The theorem is proved.

\section{IrReducible Bimodules OVER $\operatorname{Josp}(m, 2 r)$}

Recall that the Jordan superalgebra $\operatorname{Josp}(m, 2 r)$ is the superalgebra of symmetric elements of $M_{m+2 r}(F)^{(+)}$with

$$
\left(\begin{array}{ll}
a & b \\
c & d
\end{array}\right)^{\star}=\left(\begin{array}{cc}
I_{n} & 0 \\
0 & U
\end{array}\right)\left(\begin{array}{cc}
a^{t} & -c^{t} \\
b^{t} & d^{t}
\end{array}\right)\left(\begin{array}{cc}
I_{n} & 0 \\
0 & U^{-1}
\end{array}\right),
$$

where $U=\left(\begin{array}{cc}0 & -I_{r} \\ I_{r} & 0\end{array}\right)$. Therefore, $J_{\overline{0}}=\left(\begin{array}{cc}H(m) & 0 \\ 0 & H\left(M_{2 r}(F), \text { Symp }\right)\end{array}\right)$.

In this case, $\Delta(J)=\left\{0 \neq \pm w_{i} \pm w_{j}, 1 \leq i, j \leq 2 m, 0 \neq \pm v_{p} \pm v_{q}, 1 \leq p \leq q \leq\right.$ $\left.4 r, \pm w_{i} \pm v_{p}, 1 \leq i \leq 2 m, 1 \leq p \leq 4 r\right\}$. Therefore, $\Delta(J)$ coincides with the set of roots of the Lie superalgebra $K(J)$.

As in the case of $J P(n)$ - bimodules, four examples appear naturally:

(1) the regular bimodule $V=\operatorname{Josp}(m, 2 r)$,

(2) the bimodule $\operatorname{Skew}\left(M_{m+2 r}(F), \star\right)$,

(3)-(4) their opposites.

The aim now is to prove that there are no more irreducible $J$-bimodules, $J=$ $\operatorname{Josp}(m, 2 r)$.

Lemma 6.1. There are no irreducible J-bimodules $V$ such that $V J_{\overline{1}}=(0)$.

Proof. Notice first that $J_{\overline{0}}=\left[J_{\overline{1}}, J_{\overline{1}}\right]+F 1$. Indeed, $\left[J_{\overline{1}}, J_{\overline{1}}\right]$ is a subspace of $J_{\overline{0}}$ that is invariant with respect to all derivations.

For $\left(\begin{array}{ll}0 & b \\ c & 0\end{array}\right),\left(\begin{array}{cc}0 & b^{\prime} \\ c^{\prime} & 0\end{array}\right) \in J_{\overline{1}}$, we have

$$
\left[\left(\begin{array}{ll}
0 & b \\
c & 0
\end{array}\right),\left(\begin{array}{cc}
0 & b^{\prime} \\
c^{\prime} & 0
\end{array}\right)\right]=\left(\begin{array}{cc}
b c^{\prime}-b^{\prime} c & 0 \\
0 & c b^{\prime}-c^{\prime} b
\end{array}\right) .
$$

Hence the projections of the right hand side on $H(m)$ and on $H\left(M_{2 r}(F), S y m p\right)$ both may have nonzero traces. The examination of all $D\left(J_{\overline{0}}, J_{\overline{0}}\right)$-invariant subspaces of $J_{\overline{0}}$ yields that $\left[J_{\overline{1}}, J_{\overline{1}}\right]=\left\{a \in J_{\overline{0}}: \operatorname{tr}(a)=0\right\}$ and $J_{\overline{0}}=\left[J_{\overline{1}}, J_{\overline{1}}\right]+F 1$.

So $V J_{\overline{1}}=(0)$ implies that $D\left(V, J_{\overline{0}}\right)=D\left(V,\left[J_{\overline{1}}, J_{\overline{1}}\right]\right)=(0)$. In particular, $u R(x) R(a)=u R(x a)$ for arbitrary elements $u \in V, x \in J, a \in J_{\overline{0}}$.

Let $x=e_{1}, a=e_{2}$ be the identity elements of $H(m), H\left(M_{2 r}(F)\right.$, Symp), respectively. Then $V R\left(e_{1}\right) R\left(e_{2}\right)=0$ implies $V=\left\{e_{1}, V, e_{1}\right\}+\left\{e_{2}, V_{1}, e_{2}\right\}$. Both Peirce components $\left\{e_{1}, V, e_{1}\right\}$ and $\left\{e_{2}, V, e_{2}\right\}$ are $J$-bimodules; hence only one of them is not equal to zero. Let $V=\left\{e_{1}, V, e_{1}\right\}$. Then $\left\{J_{\overline{1}}, V, J_{\overline{1}}\right\} \subseteq\left\{e_{2}, V, e_{2}\right\}=(0)$. This implies $V R\left(\left[J_{\overline{1}}, J_{\overline{1}}\right]\right)=(0)$. Hence $\operatorname{dim}_{F} V=1$ and $V e_{1}=(0)$ or $V e_{2}=$ (0). But we have noticed above that the projections of $\left[J_{\overline{1}}, J_{\overline{1}}\right]$ on $H(m)$ and to $H\left(M_{2 r}(F), S y m p\right)$ both have nonzero traces, a contradiction. The lemma is proved.

Lemma 6.2. If $V$ is an irreducible J-bimodule, $J=J o s p(m, 2 r)$, then $K(V)_{w_{1}+v_{1}}$ $\neq(0)$. 
Proof. If $K(V)_{w_{1}+v_{1}}=(0)$, then the classification of irreducible $J_{\overline{0}}$-bimodules (see Chapter 2) implies that a bimodule of type iii) cannot appear. So $V=\left\{e_{1}, V, e_{1}\right\}+$ $\left\{e_{2}, V, e_{2}\right\}$. Since $J_{\overline{1}}=\left\{e_{1}, J, e_{2}\right\}$, it follows that $V J_{\overline{1}}=(0)$, which contradicts Lemma 6.1. The lemma is proved.

Let's consider a functional $\gamma: \Delta \rightarrow \mathbb{Z}$ such that $\gamma\left(w_{1}\right)>\gamma\left(v_{1}\right)>0$ and $\gamma\left(v_{1}\right)>$ $\left|\gamma\left(v_{i}\right)\right|$ if $i>1, \gamma\left(v_{1}\right)>\left|\gamma\left(w_{j}\right)\right|$ if $j>1$.

If $K(V)_{2 w_{1}} \neq(0)$, then $2 w_{1}$ is the highest weight of $K(V)$. If $K(V)_{2 w_{1}}=(0)$ and $K(V)_{w_{1}+v_{1}} \neq(0)$, then $w_{1}+v_{1}$ is the highest weight of $K(V)$. This implies

Theorem 6.3. The only finite-dimensional unital irreducible Jordan bimodules over $\operatorname{Josp}(m, 2 r)$ are the regular bimodule, the bimodule $\operatorname{Skew}\left(M_{m+2 r}(F), \star\right)$ and their opposites.

\section{Irreducible Bimodules over Jordan superalgebras of SUPERforms}

7.1. $r$-vectors. In this section we will discuss a construction which is similar to Jacobson's $r$-vectors (see [1]).

Let $V=V_{\overline{0}}+V_{\overline{1}}$ be a vector (super)space equipped with a nondegenerate supersymmetric form. We assume that $V_{\overline{1}} \neq(0)$.

Let $v_{1}, \ldots, v_{n}$ be an orthonormal basis of $V_{\overline{0}}$ and let $w_{1}, \ldots, w_{2 m}$ be a basis of $V_{\overline{1}}$ such that $\left(w_{2 i-1} \mid w_{2 i}\right)=1,1 \leq i \leq m$, where all other products are zero.

Let $C$ be the Clifford algebra of $V$. The products $v_{1}^{i_{1}} \cdots v_{n}^{i_{n}} w_{1}^{k_{1}} \cdots w_{2 m}^{k_{2 m}}$, where $0 \leq i_{1}, \ldots, i_{n} \leq 1 ; k_{1}, \ldots, k_{2 m}$ are non negative integers, form a basis of $C$.

Consider the subspace $C_{r}=\sum_{i \leq r} \underbrace{V \cdots V}_{i}$ as the span of all basic products of length $\leq r$.

$$
C_{0}=F 1 \subseteq C_{1} \subseteq C_{2} \subseteq, \cdots ; C=\bigcup_{r \geq 0} C_{r} .
$$

Let $J=F 1+V$ be the Jordan superalgebra of the superform $(\mid)$.

Lemma 7.1. If $r$ is odd, then $C_{r}$ is a J-bimodule.

Proof. Let $v, u_{1}, \ldots, u_{r} \in V_{\overline{0}} \cup V_{\overline{1}}$. We need to verify that

$$
u_{1} \cdots u_{r} v+(-1)^{\left|u_{1} \cdots u_{r}\right||v|} v u_{1} \cdots u_{r} \in C_{r} .
$$

Indeed,

$$
\begin{gathered}
u_{1} \cdots u_{r} v+(-1)^{\left|u_{1} \cdots u_{r}\right||v|} v u_{1} \cdots u_{r} \\
=\sum_{k=1}^{r}(-1)^{1+k+\left|u_{k+1} \cdots u_{r}\right||v|} u_{1} \cdots u_{k-1}\left(u_{k} v+(-1)^{\left|u_{k}\right||v|} v u_{k}\right) u_{k+1} \cdots u_{r} \in C_{r} .
\end{gathered}
$$

The lemma is proved.

If $r<0$, then we let $C_{r}=(0)$.

Lemma 7.2. For every odd $r \geq 1, C_{r} / C_{r-2}$ is an irreducible $J$-bimodule.

Proof. Let $M$ be a subbimodule of $C_{r}$, which strictly contains $C_{r-2}$.

Let $0 \neq a=\sum \alpha v_{1}^{i_{1}} \cdots v_{n}^{i_{n}} w_{1}^{k_{1}} \cdots w_{2 m}^{k_{2 m}} \in M, i_{1}, \ldots, i_{n}=0$ or $1 ; 0 \leq k_{1}, \ldots, k_{2 m}$ $\in Z$ and $i_{1}+\cdots+i_{n}+k_{1}+\cdots+k_{2 m}=r-1$ or $r$. Suppose also that $a$ has the minimal number of nonzero summands among all such elements.

For elements $x, y \in J_{\overline{0}} \cup J_{\overline{1}}$ let $D(x, y)$ denote the derivation of $C$ given by $D(x, y): c \rightarrow c\left(x y-(-1)^{|x||y|} y x\right)-(-1)^{|c||x y|}\left(x y-(-1)^{|x||y|} y x\right) c$. 
It is easy to see that

$$
v_{1}^{i_{1}} \cdots v_{n}^{i_{n}} w_{1}^{k_{1}} \cdots w_{2 m}^{k_{2 m}} D\left(w_{2 i-1}, w_{2 i}\right)=\left(k_{2 i-1}-k_{2 i}\right) v_{1}^{i_{1}} \cdots v_{n}^{i_{n}} w_{1}^{k_{1}} \cdots w_{2 m}^{k_{2 m}} .
$$

Using the Vandermonde argument and the minimal number of summands in $a$, we conclude that $k_{2 i-1}-k_{2 i}$ is the same in all summands in $a$.

Furthermore

$$
\begin{gathered}
v_{1}^{i_{1}} \cdots v_{n}^{i_{n}} w_{1}^{k_{1}} \cdots w_{2 m}^{k_{2 m}} D\left(w_{2 i-1}, w_{2 i-1}\right)^{k_{2 i}} \\
= \pm\left(k_{2 i}\right) ! v_{1}^{i_{1}} \cdots v_{n}^{i_{n}} w_{1}^{k_{1}} \cdots w_{2 i-1}^{k_{2 i-1}+k_{2 i}} w_{2 i+1}^{k_{2 i+1}} \cdots w_{2 m}^{k_{2 m}} .
\end{gathered}
$$

Applying appropriate powers of the derivations $D\left(w_{2 i-1}, w_{2 i-1}\right), 1 \leq i \leq m$, we can assume that $w_{2}, \ldots, w_{2 m}$ are not involved in the expression of $a$; that is, $k_{2}=k_{4}=\cdots=k_{2 m}=0$ in every summand.

Suppose that $i_{s} \neq 0$. Then

$$
\begin{aligned}
& v_{1}^{i_{1}} \cdots v_{n}^{i_{n}} w_{1}^{k_{1}} w_{3}^{k_{3}} \cdots w_{2 m-1}^{k_{2 m-1}} D\left(v_{s}, w_{1}\right) \\
= & \pm v_{1}^{i_{1}} \cdots v_{s-1}^{i_{s-1}} v_{s+1}^{i_{s+1}} \cdots v_{n}^{i_{n}} w_{1}^{k_{1}+1} w_{3}^{k_{3}} \cdots .
\end{aligned}
$$

Hence we can assume that in each summand $i_{1}=i_{2}=\cdots=i_{n}=0$.

If $k_{2 s-1} \geq 1, s \geq 2$, then

$$
w_{1}^{k_{1}} w_{3}^{k_{3}} \cdots w_{2 m-1}^{k_{2 m-1}} D\left(w_{2 s}, w_{1}\right)= \pm w_{1}^{k_{1}+1} w_{3}^{k_{3}} \cdots w_{2 s-1}^{k_{s}-1} \cdots .
$$

This implies that $w_{1}^{r-1} \in M$ or $w_{1}^{r} \in M$.

If $w_{1}^{r} \in M$, then $\left[w_{1}^{r}, w_{2}\right]=r w_{1}^{r-1} \in M$. If $w_{1}^{r-1} \in M$, then $w_{1}^{r}=w_{1}^{r-1} w_{1} \in M$. Hence both elements $w_{1}^{r-1}$ and $w_{1}^{r}$ lie in $M$.

Now consider an arbitrary basic element $b=v_{1}^{i_{1}} \cdots v_{n}^{i_{n}} w_{1}^{k_{1}} w_{2}^{k_{2}} \cdots w_{2 m}^{k_{2 m}}, i_{1}+\cdots+$ $i_{n}+k_{1}+\cdots+k_{2 m}=s=r-1$ or $r$. Then

$$
\begin{gathered}
w_{1}^{s} D\left(w_{2}, w_{2}\right)^{k_{2}} D\left(w_{2}, w_{3}\right)^{k_{3}+k_{4}} D\left(w_{4}, w_{4}\right)^{k_{4}} D\left(w_{2}, w_{5}\right)^{k_{5}+k_{6}} D\left(w_{6}, w_{6}\right)^{k_{6}} \\
\cdots D\left(w_{2}, v_{1}\right)^{i_{1}} D\left(w_{2}, v_{2}\right)^{i_{2}} \cdots D\left(w_{2}, v_{n}\right)^{i_{n}}
\end{gathered}
$$

is a nonzero multiple of $b$ modulo $C_{r-2}$. This proves that $M=C_{r}$. The lemma is proved.

Let $u$ be an even vector, $V^{\prime}=V+F u$. We will extend the superform to $V^{\prime}$ via $(u \mid u)=1$ and $(u \mid V)=0$.

Denote $C_{r}^{\prime}=\sum_{i \leq r} \underbrace{V^{\prime} \cdots V^{\prime}}_{i}$. If $r$ is even, then $C_{r+1}^{\prime} / C_{r-1}^{\prime}$ is an irreducible $J^{\prime}=F 1+V^{\prime}$-bimodule.

Lemma 7.3. If $r$ is even, then $u C_{r} / u C_{r-2}$ is an irreducible $J=F 1+V$-bimodule.

Proof. We have $u C_{r} \subseteq C_{r+1}^{\prime}$ and $u C_{r-2} \subseteq C_{r-1}^{\prime}$. We need to show that for arbitrary elements $x \in u C_{r} \backslash u C_{r-2}$ and $y \in u C_{r}$, there exists an operator $W$ from the multiplication algebra $U_{1}(J)$ such that $\left(x+u C_{r-2}\right) W=y+u C_{r-2}$. Since the $J^{\prime}$ bimodule $C_{r+1}^{\prime} / C_{r-1}^{\prime}$ is $J^{\prime}$-irreducible, there exists an operator $W^{\prime}$ in $U_{1}\left(J^{\prime}\right)$ such that $x W^{\prime} \in y+C_{r-1}^{\prime}$.

The operator $W^{\prime}$ is a sum of products of multiplication operators, and in each summand the element $u$ occurs an even number of times.

For an arbitrary element $v \in V, u v=0, u^{2}=1 \operatorname{imply} R(u) R(v) R(u)=0$. Hence from the Jordan identity it follows that $W^{\prime} \in \sum_{i \geq 0} R(u)^{2 i} M(J)$. But $x R(u)^{2}=x$. Hence, without loss of generality, we can assume that $W^{\prime} \in U_{1}(J)$. Now $x W^{\prime}-y \in$ $u C_{r} \cap C_{r-1}^{\prime}=u C_{r-2}$. The lemma is proved. 
7.2. Graded modules over orthosymplectic algebras. The TKK Lie superalgebra of $J$ is $L=K(J)=O S P(n+1,2 m)$. The root system of $L$ is $\Delta=$ $\left\{ \pm u_{i} \pm u_{j}, i \neq j ; \pm \omega_{p} \pm \omega_{q}, p \neq q ; \pm 2 \omega_{p} ; \pm u_{i} \pm \omega_{p} ; 1 \leq i \neq j \leq n+1 ; 1 \leq p, q \leq m\right\}$ if $n$ is odd or all the same plus $\left\{ \pm u_{i}, 1 \leq i \leq n+1\right\}$ if $n$ is even.

The short grading of $L$ which corresponds to the TKK-structure is $J^{+}=L_{1}=$ $\sum_{\left(\alpha \mid u_{1}\right)=1} L_{\alpha}, J^{-}=L_{-1}=\sum_{\left(\alpha \mid u_{1}\right)=-1} L_{\alpha},\left[J^{-}, J^{+}\right]=L_{0}=\sum_{\left(\alpha \mid u_{1}\right)=0} L_{\alpha}$.

The following elements form a basis of the Cartan subalgebra $H$ of $L_{\overline{0}}$ :

$$
h_{u_{i}}=\left(\begin{array}{cc}
\left(\begin{array}{cc}
e_{i i} & 0 \\
0 & -e_{i i}
\end{array}\right) & 0 \\
0 & 0
\end{array}\right) \quad h_{w_{j}}=\left(\begin{array}{cc}
0 & 0 \\
0 & \left(\begin{array}{cc}
e_{j j} & 0 \\
0 & -e_{j j}
\end{array}\right)
\end{array}\right)
$$

for a root element $a \in L_{\alpha},\left[h_{u_{i}}, a\right]=\left(u_{i} \mid \alpha\right) a,\left[h_{w_{j}}, a\right]=\left(w_{j} \mid \alpha\right) a$.

We remark also that $\left\langle L_{u_{i}-w_{j}}, L_{-u_{i}+w_{j}}\right\rangle=F\left(h_{u_{i}}+h_{w_{j}}\right)$.

Proposition 7.4. For an arbitrary $t \geq 2$ there exists no more than one irreducible finite-dimensional graded bimodule $M=M_{-1}+M_{0}+M_{1}$ over $L$ such that $M L_{-2 w_{1}}^{t} \neq(0), M L_{-2 w_{1}}^{t+1}=(0)$.

Proof. Let $\tilde{\Delta}$ be the set of $H$-weights of $M$. If $\lambda \in \tilde{\Delta}$, then $\left(\lambda \mid h_{u_{i}}\right) \in \mathbb{Z}$ and $\left(\lambda \mid h_{w_{j}}\right) \in \mathbb{Z}$. Hence $\lambda=\sum_{i=1}^{n+1} \alpha_{i} u_{i}+\sum_{j=1}^{m} \beta_{j} \omega_{j}, \alpha_{i}, \beta_{j} \in \mathbb{Z}$. Since $M=M_{-1}+$ $M_{0}+M_{1}$ it follows that $\alpha_{1}=-1,0,1$.

The Weyl group permutes $u_{1}, \ldots, u_{n+1}$ and for an arbitrary pair $1 \leq i \neq j \leq n+1$ the mapping $u_{i} \rightarrow-u_{i}, u_{j} \rightarrow-u_{j}, u_{k} \rightarrow u_{k}, k \neq i, j$ also lies in the Weyl group. Hence, for any weight $\lambda \in \tilde{\Delta}$ and for any $i, 1 \leq i \leq n+1$, the coefficient $\alpha_{i}$ is $-1,0,1$.

Lemma 7.5. Let $\lambda=\sum \alpha_{i} u_{i}+\sum \beta_{j} \omega_{j} \in \tilde{\Delta}, \alpha_{i}=1, \beta_{j} \geq 0$. Then $\lambda-u_{i}+\omega_{j} \in \tilde{\Delta}$.

Proof. Since $\alpha_{i}=1$, it follows that $M_{\lambda} L_{u_{i}-\omega_{j}}=(0)$. If $M_{\lambda} L_{-u_{i}+\omega_{j}}=(0)$ as well, then $M_{\lambda}\left\langle L_{u_{i}-\omega_{j}}, L_{-u_{i}+\omega_{j}}\right\rangle=(0)$, which implies $\left(\lambda \mid h_{u_{i}}+h_{\omega_{j}}\right)=0,\left(\lambda \mid u_{i}+\omega_{j}\right)=0$, $\alpha_{i}+\beta_{j}=0$, a contradiction. The lemma is proved.

The Weyl group acts on $w_{1}, \ldots, w_{m}$ by permutations and, for any $j, 1 \leq j \leq m$, contains the mapping $\omega_{j} \rightarrow-\omega_{j}, \omega_{k} \rightarrow \omega_{k}, k \neq j$.

Lemma 7.6. Let $s=\max \left\{\sum\left|\alpha_{i}\right|+\sum\left|\beta_{j}\right|: \sum \alpha_{i} u_{i}+\sum \beta_{j} \omega_{j} \in \tilde{\Delta}\right\}$. Then $s w_{1} \in \tilde{\Delta}$.

Proof. Let $\gamma=\sum \alpha_{i} u_{i}+\sum \beta_{i} \omega_{j} \in \tilde{\Delta}, \sum\left|\alpha_{i}\right|+\sum\left|\beta_{j}\right|=s$, and among all such weights the coefficient $\beta_{1}$ is maximal.

Let $\left|\alpha_{i}\right|=\max \left(\left|\alpha_{k}\right|, 1 \leq k \leq n+1\right\}$. Without loss of generality, we can assume that $\alpha_{i} \geq 0$. If $\alpha_{i} \geq 1$, then $\gamma-u_{i}+\omega_{1} \in \tilde{\Delta}$, which contradicts the maximality of $\beta_{1}$. Hence, $\alpha_{1}=\cdots=\alpha_{k+1}=0, \gamma=\sum_{i=1}^{m} \beta_{i} \omega_{i}, \sum\left|\beta_{i}\right|=s$.

Again, without loss of generality we assume that $\beta_{2} \geq 0, \ldots, \beta_{m} \geq 0$. Let $\beta_{j} \geq 1,2 \leq j \leq m$. Then $\gamma+\omega_{j}-u_{1} \notin \tilde{\Delta}$ by maximality of $s$.

Since $\left(\gamma \mid u_{1}+\omega_{j}\right) \neq 0$ it follows that $\gamma-\omega_{j}+u_{1} \in \tilde{\Delta}$. Again by Lemma 7.5 we eliminate $u_{i}$ and increase $\beta_{1}$, a contradiction. Hence, $\beta_{2}=\cdots=\beta_{m}=0, \gamma=s \omega_{1}$. The lemma is proved.

There exists a functional $f: \Lambda=\sum_{i=1}^{n+1} \mathbb{Z} u_{i}+\sum_{j=1}^{m} \mathbb{Z} \omega_{j} \rightarrow \mathbb{Z}$ such that $\pm f\left(u_{i}\right)$, $\pm f\left(\omega_{j}\right)$ are distinct and $f\left(\omega_{1}\right)=\max \left\{\left|f\left(u_{i}\right)\right|,\left|f\left(\omega_{j}\right)\right|\right\}$. This functional defines a partial order in $\Lambda$ making $s w_{1}$ the highest weight of $M$. This proves the lemma. 
Let $r$ be odd and consider the irreducible module $\bar{K}\left(C_{r} / C_{r-2}\right)$ over $L=K(J)$.

The subspace $L_{-2 \omega_{i}}$ acts on $\left(C_{r} / C_{r-2}\right)^{+}$as $D\left(\omega_{2 i}, \omega_{2 i}\right)$. We have $J D\left(\omega_{2 i}, \omega_{2 i}\right)=$ $F \omega_{2 i}, C_{r} D\left(\omega_{2 i}, \omega_{2 i}\right)^{r}=F \omega_{2 i}^{r}, C_{r} D\left(\omega_{2 i}, \omega_{2 i}\right)^{r+1}=0$. Hence $\left[\left(C_{r} / C_{r-2}\right)^{+}, J^{-}\right] L_{-2 \omega_{i}}^{r+1}$ $=F\left[\left(\omega_{2 i}^{r}\right)^{+}, \omega_{2 i}^{-}\right] \neq(0)$ in $\bar{K}\left(C_{r} / C_{r-2}\right)$, but $\bar{K}\left(C_{r} / C_{r-2}\right) L_{-2 \omega_{i}}^{r+2}=(0)$. Similarly, for an even $r$ we have $\bar{K}\left(u C_{r} / u C_{r-2}\right) L_{-2 \omega_{i}}^{r+1} \neq(0)$, but $\bar{K}\left(u C_{r} / u C_{r-2}\right) L_{-2 \omega_{i}}^{r+2}=(0)$.

Now let $B$ be an irreducible unital finite-dimensional $J$-module, $M=\bar{K}(V)$, $M L_{-2 \omega_{1}}^{s} \neq(0), M L_{-2 \omega_{1}}^{s+1}=(0), s \geq 2$. If $s$ is even, then by Proposition 7.4, $B \simeq C_{s-1} / C_{s-3}$; if $s$ is odd, then $B \simeq u C_{s-1} / u C_{s-3}$.

We proved the following theorem.

Theorem 7.7. The only finite-dimensional unital irreducible Jordan bimodules over $J=F 1+V$ are $C_{r} / C_{r-2}$ if $r$ is odd and $u C_{r} / u C_{r-2}$ if $r$ is even.

\section{JORDAN SUPERALGEBRAS OF RANK $\geq 3$}

In this chapter we consider Jordan superalgebras whose even part contains 3 pairwise orthogonal idempotents. We prove the following theorem, which extends the result by N. Jacobson [1].

Theorem 8.1. Let $J$ be a finite-dimensional simple Jordan superalgebra whose even part contains 3 pairwise orthogonal idempotents. Then its universal multiplicative enveloping algebra $U(J)$ is finite-dimensional and semisimple.

The theorem applies to Jordan superalgebras of the types $J P(n), n \geq 3 ; Q(n)^{(+)}$, $n \geq 3 ; J o s p(n, 2 m), n+m \geq 3 ; M_{m+n}^{(+)}, m+n \geq 3$, and to the exceptional Jordan superalgebra $K_{10}$.

By the result of A.S. Shtern [16], $U\left(K_{10}\right) \simeq M_{4+6}(F) \oplus M_{6+4}(F)$. Therefore, in what follows we assume that the superalgebra $J$ is special.

In [11] we have already proved the assertion for the universal special enveloping algebra $S(J)$. Now we will prove it for $U_{1}(J)$.

\subsection{Finite dimension of $U_{1}(J)$.}

Proposition 8.2. $\operatorname{dim}_{F} U_{1}(J)<\infty$.

Proof. Let $V$ be a free unital $J$-bimodule. We will denote $R(a)=R_{V}(a)$ and show that there is $m \geq 1$ such that

$$
U_{1}(J)=\langle R(J)\rangle=\sum_{k=1}^{m} \underbrace{R(J) \cdots R(J)}_{k} .
$$

This will imply that $\operatorname{dim} U_{1}(J)<(\operatorname{dim} J)^{m+1}$.

Let $d=\operatorname{dim}_{F} J_{\overline{0}}$.

We say that an operator $R\left(a_{1}\right) \cdots R\left(a_{k}\right), a_{i} \in J_{\overline{0}} \cup J_{\overline{1}}$, is irreducible if it does not lie in $\sum_{i=1}^{k-1} \underbrace{R(J) \cdots R(J)}_{i}$.

Step 1 (Jacobson). If $a_{i} \in J_{\overline{0}}, 1 \leq i \leq k$, and $R\left(a_{1}\right) \cdots R\left(a_{k}\right)$ is irreducible, then $k \leq 2 d$. Indeed, suppose that $k \geq 2 d+1$. An element

$$
R\left(a_{1}\right) \cdots R\left(a_{k}\right)+\sum_{i=1}^{k-1} \underbrace{R(J) \cdots R(J)}_{i} \in \sum_{i=1}^{k-1} \underbrace{R(J) \cdots R(J)}_{i} / \sum_{i=1}^{k-1} \underbrace{R(J) \cdots R(J)}_{i}
$$

is skew-symmetric in $d+1$ elements $a_{1}, a_{3}, a_{5}, \ldots, a_{2 d+1}$. 
Step 2. Suppose that $a_{i} \in J_{\overline{0}} \cup J_{\overline{1}}$ and the operator $R\left(a_{1}\right) \cdots R\left(a_{k}\right)$ is irreducible. Then $\left|\left\{i \mid 1 \leq i \leq k, a_{i} \in J_{\overline{0}}\right\}\right| \leq 3 d$.

If $a_{i}, a_{i+1} \in J_{\overline{0}}$ then "push" them to the left via the Jordan identity. If $a_{i}, a_{i+1} \in$ $J_{\overline{1}}$ then "push" them to the right via the Jordan identity.

We will get

$$
\begin{gathered}
R\left(a_{1}\right) \cdots R\left(a_{k}\right) \\
\in \sum R\left(b_{1}\right) \cdots R\left(b_{r}\right)\left(\prod_{i=1}^{t} R\left(x_{i}\right) R\left(c_{i}\right)\right) R\left(z_{1}\right) \cdots R\left(z_{s}\right)+\sum_{i=1}^{k-1} \underbrace{R(J) \cdots R(J)}_{i},
\end{gathered}
$$

and for each summand $r+2 t+s=k ; b_{1}, \ldots, b_{r}, c_{1}, \ldots, c_{t} \in J_{\overline{0}} ; x_{1}, \ldots, x_{t}, z_{1}, \ldots, z_{s}$ $\in J_{\overline{1}}$, and $b_{1}, \ldots, b_{r}, x_{1}, \ldots, x_{t}, c_{1}, \ldots, c_{t}, z_{1}, \ldots, z_{s}$ is a permutation of $a_{1}, \ldots, a_{k}$.

The expression $\prod_{i=1}^{t} R\left(x_{i}\right) R\left(c_{i}\right)$ is skew-symmetric in $c_{1}, \ldots, c_{t}$ modulo $\sum_{j=1}^{2 t-1} \underbrace{R(J) \cdots R(J)}_{j}$. Hence $t \leq d$. By Step $1, r \leq 2 d$. This implies the assertion.

Step 3. Let $J=\operatorname{Josp}(n, m)$ or $J P(n), n \geq 3$. Then

$$
U_{1}(J)=\sum_{k=1}^{4 d+2} \underbrace{R(J) \cdots R(J)}_{k} .
$$

Indeed, consider an irreducible operator

$$
R\left(b_{1}\right) \cdots R\left(b_{r}\right)\left(\prod_{i=1}^{t} R\left(x_{i}\right) R\left(c_{i}\right)\right) R\left(z_{1}\right) \cdots R\left(z_{s}\right) ;
$$

$b_{1}, \ldots, b_{r}, c_{1}, \ldots, c_{t} \in J_{\overline{0}} ; x_{1}, \ldots, x_{t}, z_{1}, \ldots, z_{s} \in J_{\overline{1}}$.

As we have seen above $r \leq 2 d, t \leq d$. From the identity (4) it follows that

$$
R\left(J_{\overline{1}}\right) R\left(J_{\overline{1}}\right) R\left(J_{\overline{1}}\right) \subseteq R(J)+R(J) R(J)+D\left(J_{\overline{1}}, J_{\overline{1}}\right) R\left(J_{\overline{1}}\right) .
$$

From Lemma 2.3(1) it follows that $L_{\overline{0}}=K\left(J_{\overline{0}}\right)$, hence $D\left(J_{\overline{1}}, J_{\overline{1}}\right) \subseteq R\left(J_{\overline{0}}\right)+$ $D\left(J_{\overline{0}}, J_{\overline{0}}\right)$. This shows that $s \leq 2$. The claim is proved.

From now on, $J=M_{p+q}(F)^{(+)}, p \geq q, p+q \geq 3$ or $J=Q_{n}(F)^{(+)}, n \geq 3$.

In view of the above, it is sufficient to consider only irreducible operators of the type

$$
R\left(b_{1}\right) \cdots R\left(b_{r}\right)\left(\prod_{i=1}^{t} R\left(w_{i}\right) R\left(c_{i}\right)\right) R\left(z_{1}\right) \cdots R\left(z_{\nu}\right)\left(\prod_{i=1}^{\mu} D\left(x_{i}, y_{i}\right) ;\right.
$$

$b_{1}, \ldots b_{r}, c_{1}, \ldots, c_{t} \in J_{\overline{0}} ; w_{1}, \ldots, w_{t}, z_{1}, \ldots, z_{\nu}, x_{1}, y_{1}, \ldots, x_{\mu}, y_{\mu} \in J_{\overline{1}}, \nu \leq 2, r \leq$ $2 d, t \leq d$.

Our aim is to bound the number $\mu$.

Step 4 . Let $e_{1}, \ldots, e_{n}$ be a frame of $J_{\overline{0}}$; that is, $e_{1}, \ldots, e_{n}$ are pairwise orthogonal idempotents and $n$ is maximal with this property.

Two orthogonal idempotents $e, f \in J_{\overline{0}}$ are said to be strongly connected (see [1]) if there exists an element $a_{e, f} \in\left\{e, J_{\overline{0}}, f\right\}$ such that $a_{e, f}^{2}=e+f$. In this case we denote $e \sim f$.

If $J=Q(n)^{(+)}$, then all the idempotents $e_{1}, \ldots, e_{n}$ are strongly connected. If $J=M_{p+q}(F)^{(+)}, e_{i}=e_{i i}$, then $e_{1}, \ldots, e_{p}$ are strongly connected and so are $e_{p+1}, \ldots, e_{p+q}$. 
An element $x \in J_{\overline{1}}$ is said to be Peirce homogeneous if $x \in \bigcup_{1 \leq i, j \leq n} J_{i, j}$, where $J_{i j}=\left\{e_{i}, J, e_{j}\right\}$.

Without loss of generality all the elements $x_{1}, y_{1}, \ldots, x_{\mu}, y_{\mu}$ can be assumed to be Peirce homogeneous.

Let us prove a few statements about inner derivations of Peirce homogeneous elements.

1) If $x, y \in J_{\overline{1}}$ don't lie in the same Peirce component, then $D(x, y) \in D\left(J_{\overline{0}}, J_{\overline{0}}\right)$.

Indeed, if Peirce homogeneous elements $x, y$ do not lie in the same component, then there exists $k, 1 \leq k \leq n$, such that $e_{k}$ kills one of them but does not kill any other.

Let $e_{k} \cdot x=\alpha x, \alpha=1$ or $\frac{1}{2}, e_{k} \cdot y=0$. Then $D(x, y)=D\left(\frac{1}{\alpha} e_{k} \cdot x, y\right)=D\left(\frac{1}{\alpha} e_{k}, x y\right)$ by the identity (2).

2) If $i, j, k$ are distinct and $e_{i} \sim e_{j}$, then

$$
D\left(\left(J_{\overline{1}}\right)_{i k},\left(J_{\overline{1}}\right)_{i k}\right) \subseteq D\left(\left(J_{\overline{1}}\right)_{j k},\left(J_{\overline{1}}\right)_{j k}\right)+D\left(J_{\overline{0}}, J_{\overline{0}}\right) .
$$

Indeed, since $e_{i}$ and $e_{j}$ are strongly connected, there exists $a_{i j} \in\left(J_{\overline{0}}\right)_{i j}$ such that $a_{i j}^{2}=e_{i}+e_{j}$. We have $\left\{a_{i j}, x_{i k}, a_{i j}\right\}=0$, which implies $a_{i j} \cdot\left(a_{i j} \cdot x_{i k}\right)=\frac{1}{2} a_{i j}^{2} \cdot x_{i k}=$ $\frac{1}{4} x_{i k}$.

Denote $x_{j k}^{\prime}=4 a_{i j} \cdot x_{i k}$. We have

$$
\begin{gathered}
D\left(x_{i k}, y_{i k}\right)=D\left(a_{i j} \cdot x_{j k}^{\prime}, y_{i k}\right) \\
=D\left(x_{j k}^{\prime}, a_{i j} \cdot y_{i k}\right)+D\left(a_{i j}, x_{j k}^{\prime} y_{i k}\right) \in D\left(\left(J_{\overline{1}}\right)_{j k},\left(J_{\overline{1}}\right)_{j k}\right)+D\left(J_{\overline{0}}, J_{\overline{0}}\right) .
\end{gathered}
$$

3) If $e_{i} \sim e_{j}$, then $D\left(\left(J_{\overline{1}}\right)_{i i},\left(J_{\overline{1}}\right)_{i i}\right) \subseteq D\left(\left(J_{\overline{1}}\right)_{j j},\left(J_{\overline{1}}\right)_{j j}\right)+D\left(J_{\overline{0}}, J_{\overline{0}}\right)$.

Indeed, $\left(J_{\overline{1}}\right)_{j j}=\left\{a_{i j},\left(J_{\overline{1}}\right)_{i i}, a_{i j}\right\}$ (see [1]). For two arbitrary elements $x_{i i} \in$ $\left(J_{\overline{1}}\right)_{i i}, y_{j j} \in\left(J_{\overline{1}}\right)_{j j}$ we have

$D\left(\left\{a_{i j}, x_{i i}, a_{i j}\right\}, y_{j j}\right)=D\left(2 a_{i j} \cdot\left(a_{i j} \cdot x_{i i}\right)-a_{i j}^{2} \cdot x_{i i}, y_{j j}\right)=2 D\left(a_{i j} \cdot\left(a_{i j} \cdot x_{i i}\right), y_{j j}\right)$.

Applying the identity (2) twice, we get

$$
D\left(a_{i j} \cdot\left(a_{i j} \cdot x_{i i}\right), y_{j j}\right)=D\left(x_{i i}, a_{i j} \cdot\left(a_{i j} \cdot y_{j j}\right)\right) \bmod D\left(J_{\overline{0}}, J_{\overline{0}}\right),
$$

which proves the claim.

4) If $x, y$ are Peirce homogeneous odd elements which do not lie in the same Peirce component, then

$$
R(x) R(y) \in \frac{1}{2} U(x, y)+R\left(J_{\overline{0}}\right)+D\left(J_{\overline{0}}, J_{\overline{0}}\right) .
$$

Indeed, $R(x) R(y)=\frac{1}{2}(D(x, y)+U(x, y)+R(x y))$.

Now it remains to refer to 1$)$.

$5)$ Suppose that all the idempotents $e_{1}, \ldots, e_{n}$ are strongly connected (the case $J Q(n))$. Then for arbitrary elements $x_{12}, x_{12}^{\prime} \in\left(J_{\overline{1}}\right)_{12}, y_{13}, y_{13}^{\prime} \in\left(J_{\overline{1}}\right)_{13}, z_{23}, z_{23}^{\prime} \in$ $\left(J_{\overline{1}}\right)_{23}$, we have $D\left(x_{12}, x_{12}^{\prime}\right) D\left(y_{13}, y_{13}^{\prime}\right) D\left(z_{23}, z_{23}^{\prime}\right)=\sum$ (operators each containing $\leq 5$ odd multiplications).

Indeed, $I d_{V}=\sum_{1 \leq i, j \leq n} U\left(e_{i}, e_{j}\right)$. It is sufficient to prove that each $U\left(e_{i}, e_{j}\right) D\left(x_{12}, x_{12}^{\prime}\right) D\left(y_{13}, y_{13}^{\prime}\right) D\left(z_{23}, z_{23}^{\prime}\right)$ can be represented as a sum of such operators. If $j \notin\{1,2,3\}$, then we can find $1 \leq k \neq l \leq 3$ such that $i \notin\{k, l\}$ and move the $D\left(\left(J_{\overline{1}}\right)_{k l},\left(J_{\overline{1}}\right)_{k l}\right)$ component to the left. However, $U\left(e_{i}, e_{j}\right) D\left(\left(J_{\overline{1}}\right)_{k l},\left(J_{\overline{1}}\right)_{k l}\right)=$ $(0)$.

Suppose that $i, j \in\{1,2,3\}$. If $i=j$, then we can repeat the trick with $1 \leq$ $k, l \leq 3, i \notin\{k, l\}$. 
Let $i=2, j=3$. By 4) and the identity (3),

$$
\begin{aligned}
D\left(x_{12}, x_{12}^{\prime}\right) D\left(y_{13}, y_{13}^{\prime}\right) \in & U\left(\left(J_{\overline{1}}\right)_{12},\left(J_{\overline{1}}\right)_{13}\right) R\left(\left(J_{\overline{1}}\right)_{12}\right) R\left(\left(J_{\overline{1}}\right)_{13}\right) \\
& +\sum \text { (operators containing }<4 \text { odd multiplications). }
\end{aligned}
$$

Move $D\left(z_{23}, z_{23}^{\prime}\right)$ to the right of $U\left(\left(J_{\overline{1}}\right)_{12},\left(J_{\overline{1}}\right)_{13}\right)$. However, $U\left(e_{2}, e_{3}\right) U\left(J_{12}, J_{13}\right)$ $D\left(z_{23}, z_{23}^{\prime}\right)=(0)$.

Step 5 . Now we are ready to finish the case $J=Q(n)^{(+)}, n \geq 3$.

Consider $\prod_{i=1}^{\mu} D\left(x_{i}, y_{i}\right)$; the $x_{i}, y_{i}$ are Peirce homogeneous elements; $x_{i}$ and $y_{i}$ lie in the same Peirce component. If at least 3 derivations $D\left(x_{i}, y_{i}\right)$ lie in $\bigcup_{k=1}^{n} D\left(\left(J_{\overline{1}}\right)_{k k},\left(J_{\overline{1}}\right)_{k k}\right)$, then move them to the left. By 3$)$ it is sufficient to consider

$$
D\left(\left(J_{\overline{1}}\right)_{11},\left(J_{\overline{1}}\right)_{11}\right) D\left(\left(J_{\overline{1}}\right)_{22},\left(J_{\overline{1}}\right)_{22}\right) D\left(\left(J_{\overline{1}}\right)_{33},\left(J_{\overline{1}}\right)_{33}\right),
$$

but this product is equal to 0 .

Hence $\leq 2$ factors lie in $\bigcup_{k=1}^{n} D\left(J_{k k}, J_{k k}\right)$.

Let $\mu \geq 5$. Without loss of generality we can assume that $D\left(x_{i}, y_{i}\right), 1 \leq i \leq 3$, do not lie in $\bigcup_{k=1}^{n} D\left(J_{k k}, J_{k k}\right)$. By 2$)$ it is sufficient to consider

$$
D\left(\left(J_{\overline{1}}\right)_{12},\left(J_{\overline{1}}\right)_{12}\right) D\left(\left(J_{\overline{1}}\right)_{13},\left(J_{\overline{1}}\right)_{13}\right) D\left(\left(J_{\overline{1}}\right)_{23},\left(J_{\overline{1}}\right)_{23}\right)
$$

and then use 5).

Hence we can assume $\mu \leq 4$. The case of $Q(n)^{(+)}$is finished.

Now let $J=M_{p+q}(F)^{(+)}, p \geq 2, q \geq 1$. Then $\left(J_{\overline{1}}\right)_{i j}$ is 2-dimensional, $\left(J_{\overline{1}}\right)_{i j}=$ $F \bar{e}_{i j}+F \bar{e}_{j i}$, where $\bar{e}_{i j}$ is the matrix having the element $(i, j), 1 \leq i \leq p, p+1 \leq$ $j \leq p+q$, equal to one and the rest of the elements equal to zero; the matrix $\bar{e}_{j i}$ is defined similarly.

Step 6. $D\left(\bar{e}_{i j}, \bar{e}_{i j}\right)=R_{V}\left(\bar{e}_{i j}\right)^{2}=0$, and similarly $D\left(\bar{e}_{j i}, \bar{e}_{j i}\right)=0$.

Indeed, choose $1 \leq k \leq p, k \neq i$. Then $\bar{e}_{i j}=e_{i k} \cdot \bar{e}_{k j}$ and $D\left(\bar{e}_{i j}, \bar{e}_{i j}\right)=$ $D\left(e_{i k} \cdot \bar{e}_{k j}, \bar{e}_{i j}\right)=D\left(e_{i k},\left[\bar{e}_{k j}, \bar{e}_{i j}\right]\right)+D\left(\bar{e}_{k j}, e_{i k} \cdot \bar{e}_{i j}\right)=0$.

This proves the claim.

Step 7.

$$
\begin{gathered}
D\left(\left(J_{\overline{1}}\right)_{i j},\left(J_{\overline{1}}\right)_{i j}\right)^{2}=F D\left(\bar{e}_{i j}, \bar{e}_{j i}\right)^{2} \\
=F R\left(\bar{e}_{i j}\right) R\left(\bar{e}_{j i}\right) R\left(\bar{e}_{i j}\right) R\left(\bar{e}_{j i}\right)+F R\left(\bar{e}_{j i}\right) R\left(\bar{e}_{i j}\right) R\left(\bar{e}_{j i}\right) R\left(\bar{e}_{i j}\right) \\
=F R\left(\bar{e}_{i j}\right)\left(U\left(\bar{e}_{j i}, \bar{e}_{i j}\right)+R\left(\bar{e}_{i j}\right) R\left(\bar{e}_{j i}\right)+R\left(\left[\bar{e}_{j i}, \bar{e}_{i j}\right]\right)\right) R\left(\bar{e}_{j i}\right) \\
+F R\left(\bar{e}_{j i}\right)\left(U\left(\bar{e}_{i j}, \bar{e}_{j i}\right)+R\left(\bar{e}_{j i}\right) R\left(\bar{e}_{i j}\right)+R\left(\left[\bar{e}_{i j}, \bar{e}_{i j}\right]\right)\right) R\left(\bar{e}_{i j}\right) \\
\subseteq U\left(\bar{e}_{j i}, \bar{e}_{i j}\right) R\left(J_{\overline{1}}\right) R\left(J_{\overline{1}}\right)+\sum \text { (operators containing <4 odd multiplications). }
\end{gathered}
$$

Step 8. $D\left(J_{\overline{1}}, J_{\overline{1}}\right)^{4} \subseteq$ Span of operators containing $<8$ odd multiplications.

Indeed, by part 2) of Step 4 it is sufficient to consider

$$
\begin{gathered}
D\left(\left(J_{\overline{1}}\right)_{1, p+1},\left(J_{\overline{1}}\right)_{1, p+1}\right)^{2} D\left(\left(J_{\overline{1}}\right)_{2, p+1},\left(J_{\overline{1}}\right)_{2, p+1}\right)^{2} \\
\subseteq U\left(\left(J_{\overline{1}}\right)_{1, p+1},\left(J_{\overline{1}}\right)_{1, p+1}\right) U\left(\left(J_{\overline{1}}\right)_{2, p+1},\left(J_{\overline{1}}\right)_{2, p+1}\right) \cdot\left(\sum_{i=1}^{4} R(J)^{i}\right) .
\end{gathered}
$$


Now apply identity (5) to the product of two $U$-operators and notice that

$$
V\left(\left(J_{\overline{1}}\right)_{1, p+1},\left(J_{\overline{1}}\right)_{2, p+1}\right) \subseteq R(J)+D\left(J_{\overline{0}}, J_{\overline{0}}\right)
$$

by 1 ). This proves the claim.

We showed that $\mu \leq 7$. The proposition is proved.

8.2. Complete reducibility. As above, we consider a special simple finite dimensional Jordan superalgebra of rank $\geq 3$.

Proposition 8.3. Every unital Jordan J-bimodule is completely reducible.

Part 1. $J=J P(n)$ or $J=Q(n)^{(+)}, n \geq 3$.

Let $A$ be an alternative superalgebra with a superinvolution $\sigma: A \rightarrow A$. An alternative $A$-bimodule equipped with a linear transformation $\tau: V \rightarrow V$ is called an involutive alternative bimodule over $(A, \sigma)$ if the linear transformation $A+V \rightarrow$ $A+V, a+v \rightarrow \sigma(a)+\tau(v)$ is a superinvolution of the split extension $A+V$.

In this case, $H_{n}(V, \tau)=\left\{\left(v_{i j}\right)_{1 \leq i, j \leq n} \in M_{n}(V), v_{i j} \in V, v_{j i}=\tau\left(v_{i j}\right)\right\}$ is a bimodule over $H_{n}(A, \sigma)=\left\{\left(a_{i j}\right)_{1 \leq i, j \leq n} \in M_{n}(A), a_{i j} \in A, a_{j i}=\sigma\left(a_{i j}\right)\right\}$.

The following theorem is an analog of N. Jacobson's coordinatization theorem [1].

Theorem 8.4 (see [9]). Let $J$ be a unital Jordan superalgebra, $1=\sum_{i=1}^{n} e_{i}$, where $e_{1}, \ldots, e_{n}$ are pairwise orthogonal, strongly connected idempotents of $J_{\overline{0}}$. Let $n \geq 3$ and let $V$ be a unital $J$-bimodule. Then there exists an alternative superalgebra $A$ with an involution $\sigma$ such that $H_{n}(A, \sigma) \simeq J$ and a unital involutive alternative module $(W, \tau)$ over $(A, \sigma)$ such that $V \simeq H_{n}(W, \tau)$ as an $H_{n}(A, \sigma)$-bimodule. Furthermore, the subspace of symmetric elements $H(A+W, \sigma+\tau)$ is in the associative center of $A+W$.

It is easy to see that:

(I) If $(W, \tau)$ is an irreducible involutive $(A, \sigma)$-bimodule, then $H_{n}(W, \tau)$ is an irreducible $H_{n}(A, \sigma)$-bimodule.

(II) If $(W, \tau)$ is a completely reducible $(A, \tau)$-bimodule, then $H_{n}(W, \tau)$ is a completely reducible $(A, \tau)$-bimodule.

We have

$$
\begin{gathered}
J P(n) \simeq H_{n}\left(M_{1+1}(F), \sigma\right), \quad\left(\begin{array}{ll}
a & b \\
c & d
\end{array}\right)^{\sigma}=\left(\begin{array}{cc}
d & -b \\
c & a
\end{array}\right), \\
Q(n)^{(+)} \simeq H_{n}(B, \sigma), \quad B=\left(F+F v_{1}\right) \oplus\left(F+F v_{2}\right), \quad v_{1}^{2}=1, v_{2}^{2}=-1, \\
B_{\overline{0}}=F \oplus F, \quad B_{1}=F v_{1}+F v_{2}, \quad\left(\alpha+\beta v_{1}\right)^{\sigma}=\alpha+\beta v_{2}, \quad\left(\alpha+\beta v_{2}\right)^{\sigma}=\alpha+\beta v_{1} .
\end{gathered}
$$

Lemma 8.5. Let $(W, \tau)$ be an involutive alternative bimodule over $(A, \sigma)$. If $W$ is completely reducible (as an $A$-bimodule), then $(W, \tau)$ is completely reducible as an involutive $(A, \tau)$-bimodule.

Proof. We only need to prove that $W$ is the sum (not necessarily direct) of involutive irreducible $A$-bimodules. We have $W=\sum W_{i}$, with each $W_{i}$ an irreducible $A$ bimodule. If $W_{i}^{\sigma}=W_{i}$, then $W_{i}$ is an involutive irreducible $A$-bimodule. If $W_{i}^{\sigma} \cap$ $W_{i}=(0)$, then $W_{i}+W_{i}^{\sigma}$ is an involutive irreducible bimodule. This implies the assertion. 
Pisarenko [15] proved that an alternative module over a simple finite-dimensional associative superalgebra, different from $M_{1+1}(F)$, is associative and completely reducible. The assertion is no longer true for $M_{1+1}(F)$-bimodules.

Lemma 8.6. Let $V$ be a unital alternative bimodule over the superalgebra $A=$ $M_{1+1}(F)$. Let us assume that the element $e_{21}=\left(\begin{array}{ll}0 & 0 \\ 1 & 0\end{array}\right)$ lies in the associative center of $A+V$. Then $V$ is an associative bimodule.

Proof. We have $\left[e_{21}, A\right](A+V, A+V, A+V)=(0)$ (example 6, page 144 of [19]). But $\left[e_{21}, e_{12}\right]=1$; hence $(A+V, A+V, A+V)=0$, that is, $A+V$ is associative.

If $J=J P(n), n \geq 3$, then $A=M_{1+1}(F)$. For the $e_{21}=\left(\begin{array}{ll}0 & 0 \\ 1 & 0\end{array}\right)$ we have $e_{21}^{\sigma}=e_{21}$; hence it lies in the associative center of $A+V$. Now complete reducibility of $J$-bimodules follows from Lemma 8.6.

In the case $J=Q(n)^{(+)}$all alternative modules over $B$ are associative and completely reducible by Pisarenko's result.

Part 2. $J=\operatorname{Josp}(m, 2 n)$ or $M_{m+n}(F)^{(+)}$.

In this case $J_{\overline{0}}$ is a direct sum of two simple algebras,

$$
J_{\overline{0}}=\left\{e_{1}, J_{\overline{0}}, e_{1}\right\} \oplus\left\{e_{2}, J_{\overline{0}}, e_{2}\right\}
$$

with $1=e_{1}+e_{2}$ and $J_{\overline{1}}=\left\{e_{1}, J_{\overline{1}}, e_{2}\right\}$.

We say that a unital Jordan $J$-bimodule $V$ is in the normal form if $V_{\overline{0}}=$ $\left\{e_{1}, V_{\overline{0}}, e_{1}\right\} \oplus\left\{e_{2}, V_{\overline{0}}, e_{2}\right\}, V_{\overline{1}}=\left\{e_{1}, V_{\overline{1}}, e_{2}\right\}$.

Every unital Jordan $J$-bimodule is the sum of a $J$-bimodule in the normal form and the opposite of a $J$-bimodule in the normal form.

Let $V$ be a finite-dimensional unital Jordan bimodule over $J$ in the normal form.

According to the classification of unital irreducible bimodules (Chapters 4 and 6) we know that every irreducible bimodule in the normal form has two Peirce components in the even part and the odd part is nonzero.

Consequently, for $V=V_{\overline{0}}+V_{\overline{1}}$ we have:

(1) Both Peirce components $\left\{e_{1}, V_{\overline{0}}, e_{1}\right\}$ and $\left\{e_{2}, V_{\overline{0}}, e_{2}\right\}$ are nonzero,

(2) $V_{\overline{1}} \neq(0)$.

Let's consider the subspace $S=R_{V}\left(J_{\overline{0}}\right)+D_{V}\left(J_{\overline{1}}, J_{\overline{1}}\right) \subseteq \operatorname{End}_{F}(V)$.

Lemma 8.7. If $S$ acts completely reducibly on $V$, then $V$ is a completely reducible J-bimodule.

Proof. The Peirce component $\left\{e_{1}, V_{\overline{0}}, e_{1}\right\}$ is $S$-invariant. Let $(0) \neq W \subseteq\left\{e_{1}, V_{\overline{0}}, e_{1}\right\}$ be an irreducible $S$-module.

Claim 1. Let us show that $\tilde{W}=W+W J_{\overline{1}}+W U\left(J_{\overline{1}}, J_{\overline{1}}\right)$ is a $J$-subbimodule of $V$. Indeed, $R\left(J_{\overline{1}}\right) R\left(J_{\overline{1}}\right) \subseteq D\left(J_{\overline{1}}, J_{\overline{1}}\right)+U\left(J_{\overline{1}}, J_{\overline{1}}\right)+R\left(J_{\overline{0}}\right)$. Hence

$$
\left(W J_{\overline{1}}\right) J_{\overline{1}} \subseteq W S+W U\left(J_{\overline{1}}, J_{\overline{1}}\right) \subseteq W+W U\left(J_{\overline{1}}, J_{\overline{1}}\right) \subseteq \tilde{W} .
$$

We will prove that $W R\left(J_{\overline{1}}\right) R\left(J_{\overline{0}}\right) \subseteq W R\left(J_{\overline{1}}\right)$. Indeed, if $w \in W, a \in\left\{e_{2}, J_{\overline{0}}, e_{2}\right\}$, then $D(w, a)=0$, which implies that $w R\left(J_{\overline{1}}\right) R(a)=w R\left(J_{\overline{1}} a\right) \subseteq w R\left(J_{\overline{1}}\right)$.

Now let $a \in\left\{e_{1}, J_{\overline{0}}, e_{1}\right\}$. Then $\left\{w, J_{\overline{1}}, a\right\}=(0)$, which implies that $w R\left(J_{\overline{1}}\right) R(a)$ $\subseteq w R(a) R\left(J_{\overline{1}}\right)+w R\left(J_{\overline{1}} a\right) \subseteq W R\left(J_{\overline{1}}\right)$. 
The claim follows from the following inclusions:

$$
\begin{gathered}
U\left(J_{\overline{1}}, J_{\overline{1}}\right) R\left(J_{\overline{0}}\right) \subseteq R\left(J_{\overline{0}}\right) U\left(J_{\overline{1}}, J_{\overline{1}}\right)+U\left(J_{\overline{1}}, J_{\overline{1}}\right), \\
U\left(J_{\overline{1}}, J_{\overline{1}}\right) R\left(J_{\overline{1}}\right) \subseteq R\left(J_{\overline{1}}\right) R\left(J_{\overline{1}}\right) R\left(J_{\overline{1}}\right)+R\left(J_{\overline{0}}\right) R\left(J_{\overline{1}}\right), \\
R\left(J_{\overline{1}}\right) R\left(J_{\overline{1}}\right) R\left(J_{\overline{1}}\right) \subseteq D\left(J_{\overline{1}}, J_{\overline{1}}\right)+R\left(J_{\overline{1}}\right)+R\left(J_{\overline{0}}\right) R\left(J_{\overline{1}}\right) .
\end{gathered}
$$

Claim 2. $\tilde{W}$ is an irreducible $J$-bimodule.

Indeed, $\left\{e_{1}, \tilde{W}, e_{1}\right\}=W$. If $W^{\prime}$ is a proper $J$-bimodule of $\tilde{W}$, then $W^{\prime} \cap W=(0)$; hence $W_{\overline{0}}^{\prime}=\left\{e_{2}, W_{\overline{0}}^{\prime}, e_{2}\right\}$, which contradicts property (1) of irreducible $J$-bimodules.

Let $V^{\prime}$ be the sum of all irreducible subbimodules of $V$. We have proved that $\left\{e_{1}, V_{\overline{0}}, e_{1}\right\} \subseteq V^{\prime}$ and similarly, $\left\{e_{2}, V_{\overline{0}}, e_{2}\right\} \subseteq V^{\prime}$. Since there are no nonzero unital J-bimodules whose even part is zero, we conclude that $V / V^{\prime}=(0)$, that is, $V=V^{\prime}$, which proves the lemma.

Lemma 8.8. (1) If $J=J o s p(m, 2 n)$, then $S \subseteq R_{V}\left(J_{\overline{0}}\right)+D_{V}\left(J_{\overline{0}}, J_{\overline{0}}\right)$.

(2) Let $J=M_{m+n}(F)^{(+)}$. Let's denote as $x_{i p}$ (respectively $x_{p i}$ ) the matrix whose element $(i, p)$ (respectively $(p, i))$ is equal to 1 and the rest of the elements are equal to zero, where $1 \leq i \leq m, m+1 \leq p \leq m+n$.

Let $z=\sum_{1 \leq i \leq m, m+1 \leq p \leq m+n} D_{V}\left(x_{i p}, x_{p i}\right)$. Then $S \subseteq R_{V}\left(J_{\overline{0}}\right)+D_{V}\left(J_{\overline{0}}, J_{\overline{0}}\right)+F z$.

Proof. Let $L=\tilde{K}(J)$ be the Lie superalgebra that is the universal Tits-KantorKoecher construction of $J o s p(m, 2 n)$ (orthosymplectic Lie superalgebra). Then the even part $L_{\overline{0}}$ is semisimple and $L_{\overline{0}}=J_{\overline{0}}^{-}+\left[J_{\overline{0}}^{-}, J_{\overline{0}}^{+}\right]+J_{\overline{0}}^{+}$(see [2]), which implies (1).

The universal TKK-superalgebra of $M_{m+n}(F)^{(+)}$is isomorphic to

$$
\begin{aligned}
& {\left[M_{2 m+2 n}(F)^{(-)}, M_{2 m+2 n}(F)^{(-)}\right]=\left\{\left(\begin{array}{ll}
a & b \\
c & d
\end{array}\right): a \in M_{2 m}(F),\right.} \\
& \left.b \in M_{2 m, 2 n}(F), c \in M_{2 n, 2 m}(F), d \in M_{2 n}(F), \operatorname{tr}(a)=\operatorname{tr}(d)\right\} .
\end{aligned}
$$

Furthermore, $J_{\overline{0}}^{-}+\left[J_{\overline{0}}^{-}, J_{\overline{0}}^{+}\right]+J_{\overline{0}}^{+}=\left(\begin{array}{cc}s l_{2 m} & 0 \\ 0 & s l_{2 n}\end{array}\right)$ and

$$
L_{\overline{0}}=J_{\overline{0}}^{-}+\left[J_{\overline{0}}^{-}, J_{\overline{0}}^{+}\right]+J_{\overline{0}}^{+}+F\left(\begin{array}{cc}
2 n I_{2 m} & 0 \\
0 & 2 m I_{2 n}
\end{array}\right),
$$

which implies (2) and proves the lemma.

The two previous lemmas imply complete reducibility of the unital bimodules over $\operatorname{Josp}(m, 2 n)$. With respect to the complete reducibility of unital bimodules over $M_{m+n}(F)^{(+)}$, it is only needed to prove that the action of $z$ on $V$ is diagonalizable.

Lemma 8.9. The linear transformation $z$ is diagonalizable.

Proof. It is easy to see that the linear transformations $D_{V}\left(x_{i p}, x_{p i}\right)$ and $D_{V}\left(x_{j q}, x_{q j}\right)$ commute. Consequently we only need to prove that $D_{V}\left(x_{i p}, x_{p i}\right)$ is diagonalizable.

We will prove that $D_{V}\left(x_{1, m+1}, x_{m+1,1}\right)$ is diagonalizable.

Step 1. The inner derivation $D_{J}\left(x_{1, m+1}, x_{m+1,1}\right)$ is diagonalizable since $y D_{J}\left(x_{1, m+1}, x_{m+1,1}\right)=\left[y,\left[x_{1, m+1}, x_{m+1,1}\right]\right]$ and $\left[x_{1, m+1}, x_{m+1,1}\right]=e_{11}+e_{m+1, m+1}$ is a diagonal matrix. 
Step 2. Without loss of generality we will assume that $m \geq n$. Hence, $m \geq 2$. Let us denote $V^{\prime}=\left\{e_{22}, V, e_{11}+e_{m+1, m+1}\right\}$. We claim that $V^{\prime}$ generates the $J$ bimodule $V$. Indeed, let $\bar{V}=V / V^{\prime} R\langle J\rangle$. Then $\left\{e_{22}, \bar{V}, e_{11}+e_{m+1, m+1}\right\}=(0)$, which implies $\left\{e_{22}, \bar{V}, e_{11}\right\}=\left\{e_{22}, \bar{V}, e_{m+1, m+1}\right\}=(0)$.

Since $e_{i i}$ is strongly connected to $e_{22}$ and $e_{p p}$ is strongly connected to $e_{m+1, m+1}$, for $1 \leq i \leq m, m+1 \leq p \leq m+n$, it follows that $\left\{e_{i i}, \bar{V}, e_{p p}\right\}=(0)$. Hence $\left\{e_{1}, \bar{V}, e_{2}\right\}=(0)$, where $e_{1}=e_{11}+\cdots+e_{m m}, e_{2}=e_{m+1, m+1}+\cdots+e_{m+n, m+n}$. From the classification of irreducible $J$-bimodules we conclude that $\bar{V}=(0)$.

Step 3. It suffices to prove that $D\left(x_{1, m+1}, x_{m+1,1}\right)$ acts diagonally on $V^{\prime}$.

Let $A=R_{V^{\prime}}\left(x_{1, m+1}\right)$ and $B=R_{V^{\prime}}\left(x_{m+1,1}\right)$. Hence $D\left(x_{1, m+1}, x_{m+1,1}\right)=A B+$ $B A$.

We have

$$
\begin{gathered}
A^{2}=D\left(x_{1, m+1}, x_{1, m+1}\right)=2 D\left(e_{12} \cdot x_{2, m+1}, x_{1, m+1}\right) \\
=2 D\left(e_{12},\left[x_{2, m+1}, x_{1, m+1}\right]\right)+2 D\left(x_{2, m+1}, e_{12} \cdot x_{1, m+1}\right)=0 .
\end{gathered}
$$

Similarly, $B^{2}=0$.

Furthermore, $\left\{x_{1, m+1}, V^{\prime}, x_{m+1,1}\right\}=0$. Hence,

$$
U_{V^{\prime}}\left(x_{1, m+1}, x_{m+1,1}\right)=A B-B A-R_{V^{\prime}}\left(\left[x_{1, m+1}, x_{m+1,1}\right]\right)=0,
$$

which implies that $A B-B A=R_{V^{\prime}}\left(e_{11}-e_{m+1, m+1}\right)$. Similarly,

$$
U_{V^{\prime}}\left(e_{11}-e_{m+1, m+1}\right)=2 R_{V^{\prime}}\left(e_{11}-e_{m+1, m+1}\right)^{2}-R_{V^{\prime}}\left(e_{11}+e_{m+1, m+1}\right)=0 .
$$

Hence $(A B-B A)^{2}=\frac{1}{2} R_{V^{\prime}}\left(e_{11}+e_{m+1, m+1}\right)=\frac{1}{4} I d_{V^{\prime}}$ and hence

$$
(A B+B A)^{2}=A B A B+A B B A+B A A B+B A B A=(A B-B A)^{2}=\frac{1}{4} I d_{V^{\prime}} .
$$

This implies that $D_{V^{\prime}}\left(x_{1, m+1}, x_{m+1,1}\right)=A B+B A$ is diagonalizable. The lemma is proved.

\section{ACKNOWLEDGEMENTS}

The authors are grateful to the referee for helpful remarks.

\section{REFERENCES}

1. N. Jacobson, Structure and Representation of Jordan algebras, Amer. Math. Soc., Providence, R.I., 1969. MR0251099 (40:4330)

2. V.G. Kac, Classification of simple Z-graded Lie superalgebras and simple Jordan superalgebras, Comm. in Algebra 5 (13) (1977), 1375-1400. MR0498755 (58:16806)

3. I.L. Kantor, Jordan and Lie superalgebras defined by Poisson brackets, Algebra and Analysis, 55-79, 1989. Amer. Math. Soc. Transl. Ser. (2)151, 1992. MR 1191172 (93j:17004)

4. I. Kaplansky, Superalgebras, Pacific J. of Math. 86 (1980), 93-98. MR586871)(81j:17006)

5. I. Kaplansky, Graded Jordan Algebras I, Preprint.

6. O. Loos, Jordan pairs, Lecture Notes in Mathematics, vol. 460, Springer-Verlag, Berlin-New York, 1975. MR0444721(56:3071)

7. C. Martínez and I. Shestakov, Unital irreducible bimodules over $M_{1+1}$, Preprint.

8. C. Martínez, I. Shestakov and E. Zelmanov, Jordan algebras defined by brackets, J. London Math. Soc. (2) 64, no. 2 (2001), 357-368. MR.1853456 (2002g:17045)

9. C. Martínez, I. Shestakov and E. Zelmanov, Jordan bimodules over the superalgebras $P(n)$ and $Q(n)$, Submitted

10. C. Martínez and E. Zelmanov, Lie superalgebras graded by $P(n)$ and $Q(n)$, Proc. Natl. Acad. Sci. USA 100 no.14 (2003), 8130-8137. MR1989345(2005c:17047)

11. C. Martínez and E. Zelmanov, Specializations of Simple Jordan Superalgebras, Canad. Math. Bull. 45 (4) (2002), 653-671. MR.1941232(2003k:17040) 
12. C. Martínez and E. Zelmanov, Unital bimodules over the simple Jordan superalgebras D(t), Trans. Amer. Math. Soc. 358 no.8 (2006), 3637-3649. MR2218992 (2007b:17048)

13. C. Martínez and E. Zelmanov, Representation theory of Jordan superalgebras II, Preprint.

14. Y. Medveded and E. Zelmanov, Some counterexamples in the theory of Jordan Algebras, Nonassociative Algebraic Models, Nova Science Publish., S. González and H.C. Myung eds. (1992), 1-16. MR:1189608 (93m:17017)

15. N.A. Pisarenko, The structure of alternative superbimodules, Algebra and Logic 33 no.6 (1995), 386-397. MR:1347266 (96h:17042)

16. A.S. Shtern, Representation of an exceptional Jordan superalgebra, Funktzional Anal. i Prilozhen 21 (1987), 93-94. MR911787 (89b:17009)

17. M. N. Trushina, Irreducible representations of a certain Jordan superalgebra, J. Algebra Appl. 4, no. 1 (2005), 1-14. MR2130461 (2005k:17045)

18. C.T.C. Wall, Graded Brauer groups, J. Reine Angew Math. 213 (1964), 187-199. MR0167498 $(29: 4771)$

19. K. A. Zhevlakov, A. M. Slinko, I. P. Shestakov and A. I. Shirshov, Rings that are rearly associative, Academic Press, New York, 1982. MR668355 (83i:17001)

Departamento de Matemáticas, Universidad de Oviedo, C/ Calvo Sotelo, s/n, 33007 OVIEDO, SPAIN

E-mail address: cmartinez@uniovi.es

Department of Mathematics, University of California at San Diego, 9500 Gilman Drive, La Jolla, California 92093-0112

E-mail address: ezelmano@maths.ucsd.edu 